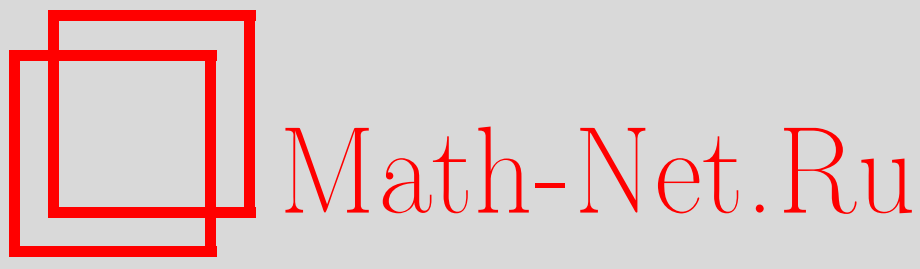

А. А. Темпельман, Размерность случайных фракталов в метрических пространствах, Теория вероятн. и ее примен., 1999, том 44, выпуск 3, 589-616

DOI: https://doi.org/10.4213/tvp805

Использование Общероссийского математического портала Math-Net.Ru подразумевает, что вы прочитали и согласны с пользовательским соглашением http://www . mathnet.ru/rus/agreement

Параметры загрузки:

IP: 18.234 .156 .22

26 апреля 2023 г., 17:00:19

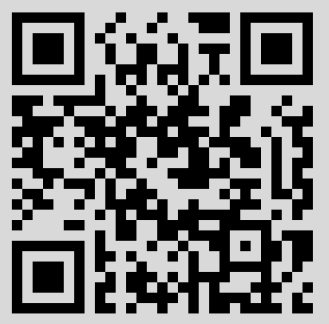




\title{
РАЗМЕРНОСТЬ СЛУЧАЙНЫХ ФРАКТАЛОВ В МЕТРИЧЕСКИХ ПРОСТРАНСТВАХ ${ }^{1)}$
}

\begin{abstract}
Изучаются локальная и хаусдорфова размерности мер в пространствах функций и последовательностей и хаусдорфова размерность таких пространств относительно детерминированных и случайных «масштабных» метрик. Вычисление хаусдорфовой размерности пространства последовательностей относительно детерминированной масштабной метрики с конечной памятью сводится к вычислению локальной размерности ассоциированной цепи Маркова; обе размерности совпадают с решением обобшенного уравнения Морана, определяемого масштабной метрикой. При случайной масштабной метрике мы приходим к стохастическому аналогу уравнения Морана. Эти результаты применены к изучению хаусдорфовых размерностей детерминированных и случайных фракталов в метрических пространствах.
\end{abstract}

Кляочевые слова и фразы: хаусдорфова размерность, хаусдорфова мера, локальная размерность, цепь Маркова, фрактал.

\section{Введение}

Основное внимание в этой статье уделено вычислению размерностей конечных мер в пространстве последовательностей и хаусдорфовой размерности самого этого пространства при заданной «масштабной» метрике; эти результаты применяются также для вычисления хаусдорфовой размерности фрактальных множеств в метрических пространствах, полученных с помощью детерминированных и случайных конструкций.

В $\S 1$ рассматривается метрическое пространство функций со значениями в конечном множестве $S$, определенных на произвольном счетном множестве $T$; определены различные понятия размерности мер и множеств в таком пространстве, и изучается связь между ними при различных масштабных условиях на метрику. Мы рассматриваем здесь два вида локальных размерностей мер - размерности, определяемые «по

\footnotetext{
*Department of Mathematics \& Department of Statistics, The Pennsylvania State University, University Park, PA 16802, USA; e-mail: arkady@stat.psu.edu

1) Работа выполнена при финансовой поддержке NSF (грант DEB-9524722).
} 
шарам», и размерности «по цилиндрическим множествам»- и устанавливаем условия их совпадения; этот результат сущцественно используется в дальнейшем. При этом основное внимание уделяется понятию локальной размерности, определяемой цилиндрическими множествами: это понятие является более естественным и удобным при изучении пространства $S^{T}$ - в отличие от конечномерного случая, где широко используется локальная размерность, определяемая шарами; понятие «цилиндрической» локальной размерности меры широко использовалось в работах Биллингсли [6]-[8] и Фюрстенберга [15]. Теорема 1.5 устанавливает связь между локальными и «глобальными» размерностями; она представляет собой модифицированную форму теоремы Биллингсли, установленной им для «цилиндрической» локальной размерности в пространстве последовательностей со стандартной метрикой, и теоремы Янг [27] для «шаровой» локальной размерности в $\mathbf{R}^{n}$. Основной результат этого параграфа - теорема 1.6 - утверждает, что при сравнительно слабых «масштабных» ограничениях на метрику все рассматриваемые размерности совпадағт. Это открывает широкие возможности для вычисления «глобальной» размерности множеств путем нахождения локальной размерности соответствующим образом подобранных мер. Этот метод восходит к цитированным выше работам Биллингсли и Фюрстенберга и широко использовался в работах других авторов.

Остальная часть работы посвящена вычислению хаусдорфовой размерности пространства последовательностей $S^{\mathbf{N}}(\S 2-4)$ и фракталов в метрических пространствах, определяемых детерминированными и случайными конструкциями (§5).

В §2 введены понятия коэффициента масштаба и различных типов масштабных метрик с конечной «памятью»в пространстве $S^{\mathbf{N}}$. Здесь же доказано существование локальной размерности любой эргодической трансляционно инвариантной вероятностной меры относительно «усреднимой» масштабной метрики; найдены формулы для вычисления локальной размерности такой меры в терминах удельной энтропии этой меры и «энтропии» коэффициентов масштаба. Этот подход обобщает идеи Биллингсли [6]--[8], Реньи [26] и Фюрстенберга [15]. Отметим также, что в различных ситуациях связь размерностных характеристик с энтропией изучалась позднее рядом авторов (см., например, [10], [14], [18], [19], [23], [25]).

В $§ 3$ вычисляется хаусдорфова размерность пространства $S^{\mathrm{N}}$ относительно данной масштабной метрики с конечной памятью. С этой метрикой ассоциирована цепь Маркова, вероятности перехода которой определяются коэффициентами масштаба; фигурирующий в ее определении параметр является решением обобщенного аналога уравнения Морана, введенного в [22]. при изучении хаусдорфовой размерности фракталов в $\mathbf{R}^{n}$. Показано, что этот параметр совпада- 
ет с локальной размерностью цепи Маркова и с хаусдорфовой размерностью пространства $S^{\mathbf{N}}$. Отметим, что в другой ситуации цепи Маркова использовались при вычислении размерности в работе $[20]$.

В $\S 4$ вычислена хаусдорфова размерность пространства $S^{\mathbf{N}}$ относительно случайной масштабной метрики без памяти. Эта размерность совпадает с решением стохастического варианта уравнения Морана.

Параграф 5 посвящен обобщению упомянутых результатов на фракталы в произвольных метрических пространствах. В первой части этого параграфа мы рассматриваем индуктивные конструкции фракталов, определяемые коэффициентами сжатия, зависящими, вообше говоря, от прошлых этапов конструкции. Вычисление размерности фрактала сводится к решению обобшенного уравнения Морана, в котором фигурирует спектральный радиус линейного оператора, определяемого коэффициентами масштаба и действуюшего в конечномерном пространстве (размерность этого пространства зависит от «памяти» масштаба). Во второй части $\S 5$ результаты $\S 4$ обобщаются на случайные конструкции фракталов в произвольных метрических пространствах. Хаусдорфова размерность случайных фракталов является решением найденного в $\$ 4$ стохастического аналога уравнения Морана (для случайных конструкций в $\mathbf{R}^{n}$ сходные результаты были получены другим методом в работах [18], [25]). Иной тип случайных конструкций был рассмотрен в работах [12] и [21]; при этом хаусдорфова размерность оказывается решением другого варианта стохастического уравнения Морана. Полученные здесь результаты в ряде аспектов обобщают результаты Кифера [18], Морана [22], Песина и Вайсса [25] и др. Например, теоремы 5.2 и 5.3 применимы в произвольных банаховых пространствах; теорема 5.4 и следствие 5.1 приложимы в метрических пространствах с некоторыми дополнительными свойствами; мы рассматриваем итеративные конструкции фракталов, в которых коэффициенты масштаба зависят от (конечного числа) предыдущих итераций.

Основные результаты работы были изложены в докладе автора на Конференции в честь юбилея проф. Александры Беллоу (Эванстон, СIIA, 23-26 октября 1997 г.).

В заключение хочу выразить глубокую благодарность Б. М. Гуревичу за подробное обсуждение рукописи статьи; его замечания способствовали ее существенному улучшению (в частности, был улучшен первоначальный вариант теоремы 1.2). Я признателен также И. П. Корнфельду за полезное обсуждение затронутых здесь вопросов и В. А. Афраимовичу, обратившему мое внимание на интересный класс фракталов, которые названы здесь «иерархическими системами планет и спутников» (см. §5). 


\section{§1. Размерности мер и множеств в пространствах функций}

Пусть $T$ - счетное множество и $T_{n}, n \in \mathbf{N}$, 一 последовательность его конечных подмножеств, причем $T_{n} \uparrow T$; пусть $S$ - конечное множество; для любой функции $x \in S^{T}$ рассмотрим в $S^{T}$ цилиндрические множества $C_{n}(x) \stackrel{\text { def }}{=}\left\{y: y \in S^{T}, y(t)=x(t)\right.$, если $\left.t \in T_{n}\right\}, n \in \mathbf{N}$; обозначим через $\mathscr{A}$ и $\mathscr{T}$, соответственно, $\sigma$-алгебру и топологию в $S^{T}$, порожденные всеми цилиндрическими множествами. Фиксируем замкнутое множество $\Lambda \subset S^{T}$. Обозначим $\widetilde{C}_{n}(x)=C_{n}(x) \cap \Lambda$; эти множества мы называем иилиндрическими множествами в $\Lambda ; \mathscr{T}_{\Lambda}$ - топология в $\Lambda$, щорожденная этими множествами.

Фиксируем число $N_{0} \in \mathbf{N}$ и последовательность положительных функщий $\left\{\beta_{n}(\cdot), n \geqslant N_{0}\right\}$ на $\Lambda$ такую, что

1) $\beta_{n}(x) \downarrow 0$ при любом $x \in \Lambda$;

2) каждая функция $\beta_{n}(x)$ постоянна на множестве $\widetilde{C}_{n}(x)$.

Кроме того, по мере надобности мы будем налагать следующие ограничения на последовательность $\beta_{n}(\cdot)$.

Пусть $\mu-$ конечная мера на $\mathscr{A}^{2}$.

( $\mu$-Р) $\mu$-регулярность последовательности $\beta_{n}$ : для $\mu$-почти всех $x \in \Lambda$

$$
\lim _{n \rightarrow \infty} \frac{\ln \left[\beta_{n+1}(x)\right]}{\ln \left[\beta_{n}(x)\right]}=1 .
$$

(СР) Сильная регулярность последовательности $\beta_{n}$ : существует положительная постоянная $\gamma<1$ такая, что при любых $n \geqslant N_{0}, x \in \Lambda$ имеем $\beta_{n+1}(x)>\gamma \beta_{n}(x)$.

Пусть $b>0$. Если $b \beta_{N_{0}}(x) \geqslant r$, определим натуральное число $l(r, x, b) \geqslant N_{0}$ следующим образом: $b \beta_{l(r, x, b)+1}(x)<r \leqslant b \beta_{l(r, x, b)}(x)$; если $b \beta_{N_{0}}(x)<r$, мы полагаем $l(r, x, b)=N_{0}$.

3 а м е ч а н и е 1.1. Пространство $S^{T}$ компактно в топологии $\mathscr{T}$. Поскольку функции $\beta_{n}(x)$ непрерывны в этой топологии, то $\beta_{n}(x) \downarrow 0$ равномерно по $x$. Поэтому при любом $r$ функция $l(r, \cdot, b)$ ограничена.

Рассмотрим в $\Lambda$ метрику $\rho$; обозначим через $B(x, r)$ шар радиуса $r$ с центром $x ;|A|$ и $[A]$ - соответственно, диаметр и замыкание множества $A \subset \Lambda$. Определенное ниже понятие «индекса Морана» $L$ было, по существу, введено Мораном при доказательстве теоремы III в [22]; там же доказано утверждение, аналогичное лемме 1.1. Наше изложение этого материала близко к [22] и [25].

Для любого натурального числа $n \geqslant N_{0}$ и любого $x \in \Lambda$ обозначим через $L_{n, x}(b)$ минимальное число $L=L(b)$, обладающее следующим свойством: существует не более чем $L_{n, x}(b)$ цилиндрических множеств

2) Мы всюду рассматриваем ненулевые меры на $\mathscr{A}$. 
$\widetilde{C}_{l\left(b \beta_{n}(x), y^{(i)}, b\right)}\left(y^{(i)}\right), y^{(i)} \in \Lambda$, таких, что $B\left(x, b \beta_{n}(x)\right) \cap \widetilde{C}_{l\left(b \beta_{n}(x), y^{(i)}, b\right)}\left(y^{(i)}\right) \neq$ $\varnothing$ и $\widetilde{C}_{l\left(b \beta_{n}(x), y^{(i)}, b\right)}\left(y^{(i)}\right) \cap \widetilde{C}_{l\left(b \beta_{n}(x), y^{(j)}, b\right)}\left(y^{(j)}\right)=\varnothing$ при $i \neq j$. Величина

$$
L(b)=\sup \left\{L_{n, x}(b): x \in \Lambda, n \geqslant N_{0}\right\}
$$

называется индексом Морана (относительно последовательности функций $\beta_{n}$ ) при постоянной $b$.

Лемма 1.1. Для любого натуральжого числа $n>N_{0} u$ любого $x \in \Lambda$ существует семейство иилиндрических множеств $\widetilde{C}_{l\left(b \beta_{n}(x), y^{(i)}, b\right)}\left(y^{(i)}\right), 1 \leqslant i \leqslant K_{n, x}$, maкoe, umo $K_{n, x} \leqslant L(b) u B\left(x, b \beta_{n}(x)\right) \subset$ $\cup_{i} \widetilde{C}_{l\left(b \beta_{n}(x), y^{(i)}, b\right)}\left(y^{(i)}\right)$.

Дока за те лг ь т в о. Яснн, что при любых $n>0$ и $x \in \Lambda$ семейство множеств $\mathscr{C}(n, x)=\left\{\widetilde{C}_{l\left(b \beta_{n}(x), y, b\right)}(y), y \in B\left(x, b \beta_{n}(x)\right)\right\}$ образует покрытие шара $B\left(x, b \beta_{n}(x)\right)$. Поскольку при любом $r$ функция $l(\cdot, r, b)$ ограничена и постоянна на цилиндрических множествах $C_{m}(y), m \geqslant N_{0}$, $y \in S^{T}$, то это семейство конечно.

Пусть $y \in B\left(x, b \beta_{n}(x)\right)$. Обозначим через $C(y)$ максимальное множество из семейства $\mathscr{C}(n, x)$, содержашее $y$. Заметим, что множества $C\left(y_{1}\right)$ и $C\left(y_{2}\right)$ либо не пересекаются, либо одно содержит другое; разумеется, последнее невозможно в силу определения множества $C(y)$. Ясно, что семейство $\left\{C(y), y \in B\left(x, b \beta_{n}(x)\right)\right\}$ является покрытием шара $B\left(x, b \beta_{n}(x)\right)$; в силу определения индекса $L(b)$ оно содержит не более чем $L(b)$ множеств. Лемма 1.1 доказана.

В дальнейшем, по мере надобности, будут рассматриваться следующие ограничения на метрику $\rho$.

(ССЦ) Скорость сжатия чилиндрических множеств: $\left|\widetilde{C}_{n}(x)\right| \leqslant$ $\beta_{n}(x), n \geqslant N_{0}, x \in \Lambda$.

(КИМ) Конечность индекса Морана: $L(b)<\infty$ при некотором $b$.

(ЖШ) Жесткость цилиндрических множеств: существует положительная постоянная $c$ такая, что при любых $n \geqslant N_{0}, x \in \Lambda$ имеем $\left|\widetilde{C}_{n}(x)\right| \geqslant c \beta_{n}(x)$.

Предложение 1.1. Если р удовлетворяет условию (ССЦ), то

1. Множество $\Lambda$ компактно относительно топологии $\mathfrak{T}$;

2. ІІІары $B(x, r)$ - открытые множества в топологии $\mathscr{T}_{\Lambda}$;

3. Множество $\Lambda$ компактно относительно $\rho$-топологии пространства $\Lambda$;

4. $\rho$-топология пространства $\Lambda$ эквивалентна топологии $\mathscr{T}_{\Lambda}$;

5. Всякое $\rho$-борелевское множество в $\Lambda$ принадлежит $\mathscr{A}$.

Д ок а з а т л ь с т в о. Утверждение 1 - следствие теоремы Тихонова. Утверждение 2 вытекает из условия (ССЦ). Отсюда следует, что $\mathscr{T}_{\Lambda}$ мажорирует $\rho$-топологию и поэтому тождественное отображение топологического пространства $(\Lambda, \mathscr{T})$ в метрическое пространство $(\Lambda, \rho)$ непрерывно; следовательно, всякое $\mathscr{T}$-замкнутое ( $\mathscr{T}$-компактное) 
множество $\rho$-замкнуто; тем самым утверждение 3 доказано. Утверждения 4 и 5 являются простыми следствиями предыдущих утверждений.

Пусть $\mathscr{K}$ - некоторый класс $\rho$-открытых множеств в $\Lambda$. Конечное или счетное покрытие множества $F \subset \Lambda$ множествами $A_{i} \subset \mathscr{K}$ называется $\delta$-покрытием, если $\left|A_{i}\right| \leqslant \delta$. Пусть $\alpha>0$. Обозначим:

$$
\begin{aligned}
H_{\delta, \mathscr{K}}^{\alpha}(F) & =\inf \left\{\sum_{i}\left|A_{i}\right|^{\alpha}:\left\{A_{i}\right\} \text { есть } \delta \text {-покрытие множества } F\right\} \\
H_{\mathscr{K}}^{\alpha}(F) & =\lim _{\delta \rightarrow 0} H_{\delta, \mathscr{K}}^{\alpha}(F) ; \quad D_{\mathscr{K}}(F)=\inf \left\{\alpha: H_{\mathscr{X}}^{\alpha}(F)=0\right\} .
\end{aligned}
$$

В дальнейшем в качестве $\mathscr{K}$ мы рассмотрим классы $\mathscr{U}, \mathscr{B}, \mathscr{C}$, соответственно, всех открытых множеств, всех шаров с центрами в $F$ и всех цилиндрических множеств $\widetilde{C}_{n}(x), n \geqslant N_{0}, x \in \Lambda$, в $\Lambda$. Известно, что при лююбом $\alpha$ мы имеем: $H_{\mathscr{U}}^{\alpha}(F) \leqslant H_{\mathscr{B}}^{\alpha}(F) \leqslant 2^{\alpha} H_{\mathscr{U}}^{\alpha}(F)$ (см., например, [11]). Число $D_{H}(F) \stackrel{\text { def }}{=} D_{\mathscr{U}}(F)=D_{\mathscr{B}}(F)$ называется хаусдорфовой размерностью множества $F ; H^{\alpha}(F) \stackrel{\text { def }}{=} H_{\mathscr{U}}^{\alpha}(F)$ называется хаусдорфовой мерой множестөа $F$. Если $H^{\alpha_{0}}(F)>0$ при $\alpha_{0}=D_{H}(F)$, говорят, что размерность $D_{H}(F)$ точна.

Рассмотрим также функцию множеств

$$
\widehat{H}_{\mathscr{C}}^{\alpha}(F)=\lim _{n \rightarrow \infty} \widehat{H}_{n, \mathscr{C}}^{\alpha}(F)
$$

где при любом $n \geqslant N_{0}$

$$
\begin{aligned}
\widehat{H}_{n, \mathscr{C}}^{\alpha}(F)=\inf \{ & \sum_{i}\left(\beta_{n_{i}}\left(x^{(i)}\right)\right)^{\alpha}: n_{i} \geqslant n, \\
& \left.\left\{C_{n_{i}}\left(x^{(i)}\right)\right\} \text { есть покрытие множества } F\right\},
\end{aligned}
$$

и размерность $\widehat{D}_{\mathscr{C}}(F)=\inf \left\{\alpha: \widehat{H}_{\mathscr{C}}^{\alpha}(F)=0\right\}$.

Теорема 1.1. Функция $\widehat{H}_{\mathscr{C}}^{\alpha}(F)$ является мерой на $\mathscr{A}$.

Доказательство. Нетрудно видеть, что в определении $H_{n, \mathscr{C}}^{\alpha}(F)$ можно ограничиться покрытиями, состояшими из непересекающихся щилиндрических множеств. Отсюда вытекает, что $\widehat{H}_{\mathscr{C}}^{\alpha}(F)$ адцитивна на алгебре $\mathscr{C}_{0}$ всех объединений цилиндрических множеств. Поскольку цилиндрические множества открыты и $\Lambda$ компактно в топологии $\mathscr{T}_{\Lambda}$, то функция $\hat{H}_{\mathscr{C}}^{\alpha}(F) \sigma$-аддитивна на $\mathscr{C}_{0}$, а следовательно, и на $\mathscr{A}$. Теорема 1.1 доказана.

С помощью стандартных рассуждений (см., например, [11]) нетрудно показать, что 1) $\widehat{D}_{\mathscr{C}}\left(F_{1}\right) \leqslant \widehat{D}_{\mathscr{C}}\left(F_{2}\right)$, если $\left.F_{1} \subset F_{2}, 2\right) \widehat{D}_{\mathscr{C}}\left(\cup_{i=1}^{\infty} F_{i}\right)=$ $\sup _{1 \leqslant i \leqslant \infty} \widehat{D}_{\mathscr{C}}\left(F_{i}\right)$ и 3) $\widehat{D}_{\mathscr{C}}\left(F_{n}\right) \uparrow \widehat{D}_{\mathscr{C}}(F)$, если $F_{n} \uparrow F$; отсюда вытекает, что $\widehat{D}_{\mathscr{C}}(F)=0$ для всякого счетного множества $F$. 
Теорема 1.2. Если выполнено условие (ССЦ), то $H_{\mathscr{C}}^{\alpha}(F) \leqslant$ $\widehat{H}_{\mathscr{C}}^{\alpha}(F)$ для любого множества $F \subset \Lambda$ и для любого $\alpha>0$, откуда cледует, что $D_{\mathscr{C}}(F) \leqslant \widehat{D}_{\mathscr{C}}(F)$; если, кроме (ССЦ), выполнено условие (ЖЦ), mo $H_{\mathscr{C}}^{\alpha}(F)=c^{\alpha} \widehat{H}_{\mathscr{C}}^{\alpha}(F), \alpha>0$, n nоэтомy $D_{\mathscr{C}}(F)=\widehat{D}_{\mathscr{C}}(F)$.

Д ок а затель ство. Прежде всего заметим, что функции $\left|\widetilde{C}_{n}(x)\right|$ непрерывны относительно топологии $\mathscr{T}_{\Lambda}$ и поэтому, ввиду условия (ССЩ) и компактности пространства $\Lambda,\left|\widetilde{C}_{n}(x)\right| \rightarrow 0$ при $n \rightarrow \infty$ равномерно относительно $x \in \Lambda$. Таким образом, $\left|\widetilde{C}_{n}(x)\right| \leqslant \delta$ при $n>N(\delta)$ для любых $x$ и $\delta>0$. Из условия (ССЩ) вытекает: $\widehat{H}_{n, \mathscr{C}}^{\alpha}(F) \geqslant H_{\delta, \mathscr{C}}^{\alpha}(F)$ при $n>N(\delta)$ и, таким образом, $H_{\delta, \mathscr{C}}^{\alpha}(F) \leqslant \lim _{n \rightarrow \infty} \widehat{H}_{n, \mathscr{C}}^{\alpha}(F)$. Отсюда следует, что $H_{\mathscr{C}}^{\alpha}(F) \leqslant \widehat{H}_{\mathscr{C}}^{\alpha}(F)$. Рассмотрим функцию $n=\psi(x, \delta) \stackrel{\text { def }}{=}$ $\min \left\{k: k \in \mathbf{N},\left|\widetilde{C}_{k}(x)\right| \leqslant \delta\right\}$. При любом $\delta$ функция $\psi(\cdot, \delta)$ постоянна на цилиндрических множествах и, следовательно, непрерывна в топологии $\mathscr{T}$. В силу компактности пространства $S^{T}, \psi(x, \delta) \rightarrow \infty$ при $\delta \rightarrow 0$ равномерно по $x \in S^{T}$. Поэтому пля всякого $N \geqslant 1$ существует $\delta(N)>0$ такое, что при любом $x \in \Lambda$ имеем: если $\left|\widetilde{C}_{n}(x)\right| \leqslant \delta(N)$, то $n \geqslant N$; из условия (ЖЦ) вытекает, что $H_{\delta(N), \mathscr{C}}^{\alpha}(F) \geqslant c^{\alpha} \widehat{H}_{N, \mathscr{C}}^{\alpha}(F)$ и, следовательно, $c^{\alpha} \widehat{H}_{\mathscr{C}}^{\alpha}(F) \leqslant H_{\mathscr{C}}^{\alpha}(F)$. Тем самым утверждение доказано.

Теорема 1.3. Предположим, ито выполнены условия (ССЦ) $u$ (CP). Тогда при любых F $\subset \Lambda, \alpha>0 u b>0$

$$
H^{\alpha}(F) \leqslant H_{\mathscr{C}}^{\alpha}(F) \leqslant L(b)(b \gamma)^{-\alpha} H^{\alpha}(F) .
$$

Поэтому $D_{\mathscr{C}}(F)=D_{H}(F)$.

Д о к а з а т е л ь т в о. Поскольку $\mathscr{C} \subset \mathscr{U}$, ясно, что $H_{\delta, \mathscr{U}}^{\alpha}(F) \leqslant$ $H_{\delta, \mathscr{C}}^{\alpha}(F)$ и поэтому

$$
H_{\mathscr{U}}^{\alpha}(F) \leqslant H_{\mathscr{C}}^{\alpha}(F)
$$

Фиксируем $\delta>0$; пусть $\left\{B\left(x^{(i)}, r_{i}\right)\right\}$ является $\delta$-покрытием множества $F$ шарами. Положим $n_{i}=l\left(r_{i}, x^{(i)}, b\right)$. Согласно лемме 1.1 , существуют множества $\widetilde{C}_{n_{i, k}}\left(x^{(i, k)}\right), 1 \leqslant k \leqslant K_{i}$, такие, что $K_{i} \leqslant L(b), B\left(x^{(i)}\right.$, $\left.b \beta_{n_{i}}\left(x^{(i)}\right)\right) \subset \cup_{k} \widetilde{C}_{n_{i, k}}\left(x^{(i, k)}\right)$ и $b \beta_{n_{i, k}+1}\left(x^{i, k}\right)<r_{i}$. Поэтому, в силу условия (CCII), $\left|\widetilde{C}_{n_{i, k}+1}\left(x^{(i, k)}\right)\right|<\beta_{n_{i, k}+1}\left(x^{(i, k)}\right)<b^{-1} r_{i}$. Обозначим: $\psi=(2 b \gamma)^{-1}$. Из условия (CР) вытекает:

$$
\left|\widetilde{C}_{n_{i, k}}\left(x^{(i, k)}\right)\right| \leqslant \gamma^{-1}\left|\widetilde{C}_{n_{i, k}+1}\left(x^{(i, k)}\right)\right| \leqslant 2 \psi r_{i} \leqslant \psi \delta .
$$

Таким образом, $\left\{\widetilde{C}_{n_{i}}\left(x^{(i, k)}\right)\right\}$ является $\psi \delta$-покрытием множества $F$ и

$$
\sum_{i, k}\left|\widetilde{C}_{n_{i, k}}\left(x^{(i, k)}\right)\right|^{\alpha}<L(b) \psi^{\alpha} \sum_{i}\left(2 r_{i}\right)^{\alpha} .
$$

Отсюда вытекает, что $H_{\psi \delta, \mathscr{C}}^{\alpha}(F) \leqslant L(b) \psi^{\alpha} H_{\delta, \mathscr{B}}^{\alpha}(F)$. Положим $\delta \rightarrow 0$; поскольку $H_{\mathscr{B}}^{\alpha}(F) \leqslant 2^{\alpha} H_{\mathscr{U}}^{\alpha}(F)$, мы получаем:

$$
H_{\mathscr{C}}^{\alpha}(F) \leqslant L(b) \psi^{\alpha} H_{\mathscr{B}}^{\alpha}(F) \leqslant 2^{\alpha} L(b) \psi^{\alpha} H_{\mathscr{U}}^{\alpha}(F) .
$$


Остается воспользоваться неравенствами (1.2) и (1.3). Теорема 1.3 доказана.

Пусть $\mu-$ конечная мера на $\mathscr{A}$. Локальная размерность этой меры в точке $x \in \Lambda$ определяется следуюшей формулой (в предположении, что рассматриваемый предел суцествует и конечен):

$$
d_{\mathscr{B}, \mu}(x) \stackrel{\text { def }}{=} \lim _{r \rightarrow 0} \frac{\ln \mu(B(x, r))}{\ln r} .
$$

Наряду с этой «шаровой» локальной размерностью мы рассмотрим «цилиндрическую» локальную размерность меры $\mu$ в точке $x \in \Lambda$, определяемую следующим образом (в предположении, что предел существует и конечен):

$$
d_{\mathscr{C}, \mu}(x) \stackrel{\text { def }}{=} \lim _{n \rightarrow \infty} \frac{\ln \mu\left(C_{n}(x)\right)}{\ln \left[\beta_{n}(x)\right]} .
$$

Следуюшая теорема устанавливает связь между этими понятиями локальной размерности.

Теорема 1.4. Пусть $F \in \mathscr{A}, F \subset \Lambda u \mu(F)=\mu\left(S^{T}\right)$. IIредnoложим также, что выполнены условия (ССЦ), (КИМ) $u(\mu-\mathrm{P})$. Если $\mu$-n.в. на $F$ существует $d_{\mathscr{C}, \mu}(x)$, то $\mu$-п.в. на $F$ существует $u d_{\mathscr{B}, \mu}(x)$, причем $d_{\mathscr{B}, \mu}(x)=d_{\mathscr{C}, \mu}(x) \mu$-n.6.

Д о к а з а т е л ь с т в о. Обозначим через $\widetilde{F}$ подмножество множества $F$, на котором существует $d_{\mathscr{C}, \mu}(x)$ и выполнено (1.1). Согласно нашему предположению, $\mu(\widetilde{F})=\mu(F)$. Фиксируем произвольную функцию $x(\cdot) \in \widetilde{F}$. Обозначим $r_{n}=\beta_{n}(x)$. Согласно условию (ССЦ), $\widetilde{C}_{n}(x) \subset B\left(x, r_{n}\right)$. Имеем:

$$
\limsup _{n \rightarrow \infty} \frac{\ln \mu\left(B\left(x, r_{n}\right)\right)}{\ln r_{n}} \leqslant \lim _{n \rightarrow \infty} \frac{\ln \mu\left(\widetilde{C}_{n}(x)\right)}{\ln \left[\beta_{n}(x)\right]}=d_{\mathscr{C}, \mu}(x) .
$$

Пусть $r_{n+1} \leqslant r \leqslant r_{n}$. Из условия $\left(\mu\right.$-Р) следует: $\ln r_{n} \sim \ln r_{n+1} ;$ при достаточно большом $n$

$$
\frac{\ln \mu(B(x, r))}{\ln r} \leqslant \frac{\ln \mu(B(x, r))}{\ln r_{n}} \leqslant \frac{\ln r_{n+1}}{\ln r_{n}} \frac{\ln \mu\left(B\left(x, r_{n+1}\right)\right)}{\ln r_{n+1}} .
$$

Отсюда вытекает, что

$$
\limsup _{r \rightarrow 0} \frac{\ln \mu(B(x, r))}{\ln r} \leqslant d_{\mathscr{G}, \mu}(x) .
$$

Положим теперь $r_{n}=\boldsymbol{b} \beta_{n}(x)$. Поскольку $\lim _{n \rightarrow \infty} l\left(r_{n}, y, b\right)=\infty$, для любого $y \in \widetilde{F}$ имеем: $\lim _{n \rightarrow \infty} \ln \left[\mu\left(C_{l\left(r_{n}, y, b\right)}(y)\right)\right] / \ln \left[b \beta_{l\left(r_{n}, y, b\right)}(y)\right]=d_{\mathscr{C}, \mu}$. Пусть $\dot{\varepsilon}>0, N \in \mathbf{N}$; обозначим через $\widetilde{F}_{N}^{\varepsilon}$ множество, состоящее 
функций $y \in \tilde{F}$ таких, что при $n \geqslant N, y \in \tilde{F}_{N}^{\varepsilon}$ выполнено соотношение $\ln \left[L(b) \mu\left(C_{l\left(r_{n}, y, b\right)}(y)\right)\right] / \ln \left[b \beta_{l\left(r_{n}, y, b\right)}(y)\right] \geqslant d_{\mathscr{C}, \mu}-\varepsilon$. Согласно лемме 1.1 , для любого шара $B\left(x, r_{n}\right)$ существуют цилиндрические множества $C_{l\left(r_{n}, x_{n, i}, b\right)}\left(x_{n, i}\right), 1 \leqslant i \leqslant k_{n} \leqslant L(b)$ такие, что $B\left(x, r_{n}\right) \subset$ $\cup_{i=1}^{k_{n}} C_{l\left(r_{n}, x_{n, i}, b\right)}\left(x_{n, i}\right)$. Из условия теоремы вытекает, что $\mu\left(C_{n}(x)\right)>0$ при любых $n \in \mathbf{N}$ и $x \in \tilde{F}$. Очевидно, при любом $\varepsilon>0$ мы имеем $\widetilde{F}_{N}^{\varepsilon} \uparrow \widetilde{F}$ и поэтому $\mu\left(\widetilde{F}_{N}^{\varepsilon}\right) \uparrow \mu(\widetilde{F})=\mu\left(S^{\mathbf{N}}\right)$. Далее, $\mu\left(C_{l\left(r_{n}, x_{n, i}, b\right)}\left(x_{n, i}\right) \cap\right.$ $\widetilde{F})-\mu\left(C_{l\left(r_{n}, x_{n, i}, b\right)}\left(x_{n, i}\right) \cap \widetilde{F}_{N}^{\varepsilon}\right) \leqslant \mu(\widetilde{F})-\mu\left(\widetilde{F}_{N}^{\varepsilon}\right)$ при любых $n \in \mathbf{N}, 1 \leqslant i \leqslant k_{n}$. Таким образом, если $N$ достаточно велико, то при всех $n \in \mathbf{N}, i \leqslant k_{n}$ множества $\widetilde{F}_{N}^{\varepsilon} \cap C_{l\left(r_{n}, x_{n, i}, b\right)}\left(x_{n, i}\right)$ непусты, и мы можем считать, что $x_{n, i} \in \tilde{F}_{N}^{\varepsilon}$. Пусть $x_{n}^{*} \in \widetilde{F}_{N}^{\varepsilon}$ и $\mu\left(C_{l\left(r_{n}, x_{n}^{*}, b\right)}\left(x_{n}^{*}\right)\right)=\max _{1 \leqslant i \leqslant k_{n}} \mu\left(C_{l\left(r_{n}, x_{n, i}, b\right)}\left(x_{n, i}\right)\right)$. Заметим, что $b \beta_{l\left(\tau_{n}, x_{n}^{*}, b\right)+1}\left(x_{n}^{*}\right)<r_{n}$. Поскольку $x_{n}^{*} \in \tilde{F}_{N}^{\varepsilon}$, то при любом $n>N$

$$
\frac{\ln \mu\left(B\left(x, r_{n}\right)\right)}{\ln r_{n}} \geqslant \frac{\ln \left[L(b) \mu\left(C_{l\left(r_{n}, x_{n}^{*}, b\right)}\left(x_{n}^{*}\right)\right)\right]}{\ln \left[b \beta_{l\left(r_{n}, x_{n}^{*}, b\right)+1}\left(x_{n}^{*}\right)\right]} \geqslant d_{\mathscr{C}, \mu}-\varepsilon .
$$

Так как $\widetilde{F}_{\varepsilon, N} \uparrow \tilde{F}$ при $N \rightarrow \infty$ и число $\varepsilon$ выбрано произвольно, то $\lim \inf _{n \rightarrow \infty}\left(\ln \mu\left(B\left(x, r_{n}\right)\right) / \ln r_{n}\right) \geqslant d_{\mathscr{C}, \mu} ;$ рассуждая, как и при доказательстве формулы (1.5), убеждаемся, что $\operatorname{lim~inf~}_{r \rightarrow 0}(\ln \mu(B(x, r)) / \ln r) \geqslant d_{\mathscr{C}, \mu}$. Ввиду соотношения (1.5), теорема доказана.

Перейдем к изучению связи локальных размерностей с размерностями множеств.

Следующая теорема представляет собой модифицированную форму утверждений, доказанных Биллингсли [8] и Янг [27].

Теорема 1.5. Предположим, что выполнено условие (ССЦ). Пусть $\mu$ - конечная мера на $\mathscr{A}, F \in \mathscr{A}, F \subset \Lambda$, причем $\mu(F)>0$. Предположим, что существуют постоянные $\underline{d}$ u $\bar{d}$ такие, ито $0 \leqslant \underline{d} \leqslant$ $\bar{d}<\infty$ и при любом $x \in F$

$$
\underline{d} \leqslant \liminf _{n \rightarrow \infty} \frac{\ln \mu\left(C_{n}(x)\right)}{\ln \left[\beta_{n}(x)\right]} \leqslant \limsup _{n \rightarrow \infty} \frac{\ln \mu\left(C_{n}(x)\right)}{\ln \left[\beta_{n}(x)\right]} \leqslant \bar{d} .
$$

Тогда $\underline{d} \leqslant \widehat{D}_{\mathscr{C}}(F) \leqslant \bar{d} . \quad$ В частности, если локальная размерность $d_{\mathscr{C}, \mu}(x)$ сущестөует всюду на $F$ и постоянна: $d_{\mathscr{C}, \mu}(x) \equiv d_{\mathscr{C}, \mu}$, то $\widehat{D}_{\mathscr{C}}(F)=d_{\mathscr{C}, \mu}$.

Д ок а за т л в с т в о. Пусть $\Delta-$ подмножество $\mu$-атомов в $F$. Если $\Delta \neq \varnothing$, то $\underline{d}=0$. Обозначим $F^{\prime}=F \backslash \Delta$. Поскольку $\widehat{D}_{\mathscr{C}}(\Delta)=0$, то $\widehat{D}_{\mathscr{C}}(F)=\widehat{D}_{\mathscr{C}}\left(F^{\prime}\right)$. Таким образом, достаточно доказать теорему для множества $F^{\prime}$. Мы сразу предполагаем, что мера $\mu$ неатомическая.

1. Пусть $G \in \mathscr{A}, G \subset \Lambda$ и $\mu(G)>0$; предположим, что сушествуют вешественные числа $\underline{d}^{\prime} \leqslant \bar{d}^{\prime}$ и натуральное число $N$ такие, что для любого $x \in G$ и для любого $n \geqslant N$

$$
\underline{d}^{\prime} \leqslant \frac{\ln \mu\left(C_{n}(x)\right)}{\ln \left[\beta_{n}(x)\right]} \leqslant \bar{d}^{\prime} .
$$


Рассмотрим разбиение $S^{T}=\cup_{i=1}^{m_{n}} C_{n_{i}}\left(x^{(i)}\right)$ с $n_{i} \geqslant n, 1 \leqslant i \leqslant m_{n}$. При любом $\alpha>1$

$$
\sum_{i=1}^{m_{n}}\left[\mu\left(C_{n_{i}}\left(x^{(i)}\right)\right)\right]^{\alpha} \leqslant \max _{1 \leqslant i \leqslant m_{n}}\left[\mu\left(C_{n_{i}}\left(x^{(i)}\right)\right)\right]^{\alpha-1} \mu\left(S^{T}\right)
$$

Поскольку пространство $\dot{S}^{T}$ компактно и при любом $n \geqslant 1$ функция $\mu\left(C_{n}(x)\right)$ непрерывна, то $\mu\left(C_{n}(x)\right) \downarrow 0$ при $n \uparrow \infty$ равномерно по $x$. Поэтому мы имеем:

$$
\lim _{n \rightarrow \infty} \sum_{i}\left[\mu\left(C_{n_{i}}\left(x^{(i)}\right)\right)\right]^{\alpha}=0
$$

Из второто неравенства в формуле (1.7) вытекает: $\left(\beta_{n_{i}}\left(x^{(i)}\right)\right)^{\bar{d}^{\prime}} \leqslant$ $\mu\left(C_{n_{i}}\left(x^{(i)}\right)\right)$ и, ввиду $(1.8), \widehat{H}_{\mathscr{C}}^{\bar{d}^{\prime} \alpha}(G)=0$. Таким образом, $\widehat{D}_{\mathscr{C}}(G) \leqslant \bar{d}^{\prime} \alpha$, и, поскольку $\alpha>1$ выбрано произвольно, $\widehat{D}_{\mathscr{C}}(G) \leqslant \bar{d}^{\prime}$. В силу первого неравенства в $(1.7)$, для любого покрытия $\left\{C_{n_{i}}\left(x^{(i)}\right)\right\}$ множества $G$ с индексами $n_{i}>N$

$$
\sum_{i}\left(\beta_{n_{i}}\left(x^{(i)}\right)\right)^{\underline{d}^{\prime}} \geqslant \sum_{i} \mu\left(C_{n_{i}}\left(x^{(i)}\right)\right) \geqslant \mu(G)>0 .
$$

Из этого вытекает, что $\widehat{H}_{\mathscr{C}}^{\underline{d}^{\prime}}(G)>0$ и, следовательно, $\underline{d}^{\prime} \leqslant \widehat{D}_{\mathscr{C}}(G)$. Таким образом, $\underline{d}^{\prime} \leqslant \widehat{D}_{\mathscr{C}}(G) \leqslant \bar{d}^{\prime}$.

2. Для любого $N \in \mathbf{N}$ и любого $\varepsilon>0$ рассмотрим множество

$$
F_{N}^{\varepsilon}=\bigcup_{n=N}^{\infty}\left\{x: x \in F, \underline{d}-\varepsilon \leqslant \frac{\ln \mu\left(C_{n}(x)\right)}{\ln \left[\beta_{n}(x)\right]} \leqslant \bar{d}+\varepsilon\right\} .
$$

Легко видеть, что $F=\uparrow \lim _{N \rightarrow \infty} F_{N}^{\varepsilon}$. Ясно также, что множества $F_{N}^{\varepsilon}$ измеримы и $\lim _{N \rightarrow \infty} \mu\left(F_{N}^{\varepsilon}\right)=\mu(F)$. Выше мы доказали, что $\underline{d}-\varepsilon \leqslant$ $\widehat{D}_{\mathscr{C}}\left(F_{N}^{\varepsilon}\right) \leqslant \bar{d}+\varepsilon$, коль скоро $\mu\left(F_{N}^{\varepsilon}\right)>0$. Из сказанного вытекает, что $\widehat{D}_{\mathscr{C}}(F)=\lim _{N \rightarrow \infty} \widehat{D}_{\mathscr{C}}\left(F_{N}^{\varepsilon}\right)$ и, следовательно, $\underline{d}-\varepsilon \leqslant \widehat{D}_{\mathscr{C}}(F) \leqslant \bar{d}+\varepsilon$. Поскольку $\varepsilon$ - произвольное положительное число, наше утверждение доказано.

Пример 6.8 в работе [16] показывает, что, по крайней мере, второе неравенство в теореме 1.3 теряет силу даже в простейших случаях, если $\gamma=0$. Тем не менее, равенства $D_{\mathscr{C}}(F)=\hat{D}_{\mathscr{C}}(F)=D_{H}(F)$ справедливы, при некоторых слабых ограничениях, и в этом случае. Следуюшая теорема - шаг в этом направленик.

Теорема 1.6. Пусть $\mu$ - конечная мера на $\mathscr{A}$, и пусть выполнены условия (ССЦ), (КИМ) $u$ ( $\mu$-Р). Предположим, что $F \in \mathscr{A}$, $\mu(F)=\mu\left(S^{T}\right)$ и что всюду на $F$ определена размерность $d_{\mathscr{C}, \mu}(x) \equiv d$. Tогда $D_{H}(F)=D_{\mathscr{C}}(F)=\widehat{D}_{\mathscr{C}}(F)=d$. 
Д ок а за те л ь с т о. Как и при доказательстве теоремы 1.3, имеем $H_{\mathscr{U}}^{\alpha}(F) \leqslant H_{\mathscr{C}}^{\alpha}(F)$, и поэтому

$$
D_{H}(F) \leqslant D_{\mathscr{C}}(F)
$$

Далее, согласно теореме $1.5, d=\widehat{D}_{\mathscr{C}}(F)$, а по теореме $1.4 d_{\mathscr{B}, \mu}=d$ на множестве $\widetilde{F}$ с $\mu(\widetilde{F})=\mu\left(S^{T}\right)$. Фиксируем $N>N_{0}$. Рассмотрим множество $\tilde{F}_{N}^{\varepsilon} \subset \widetilde{F}$, на котором $\ln [\mu(B(x, r))] / \ln |B(x, r)| \geqslant d-\varepsilon$ при любом $r \leqslant N^{-1}$. При $\delta<N^{-1}$ для любото $\delta$-покрытия множества $\widetilde{F}_{N}^{\varepsilon}$ шарами $\left\{B\left(x_{i}, r_{i}\right)\right\}$ с $x_{i} \in \widetilde{F}_{N}^{\varepsilon}$ имеем: $\sum_{i}\left(\left|B\left(x_{i}, r_{i}\right)\right|\right)^{d-\varepsilon} \geqslant \sum_{i} \mu\left(B\left(x_{i}, r_{i}\right)\right) \geqslant$ $\mu\left(\widetilde{F}_{N}^{\varepsilon}\right)$, где $\mu\left(\widetilde{F}_{N}^{\varepsilon}\right)>0$ при достаточно больших $N$. Отсюда следует, что для таких $N D_{H}\left(F_{N}^{\epsilon}\right) \geqslant d-\varepsilon$. Очевидно, $\widetilde{F}=\uparrow \lim _{N \rightarrow \infty} \widetilde{F}_{N}^{\varepsilon}$, и поэтому $D_{H}(\widetilde{F})=\lim _{N \rightarrow \infty} D_{H}\left(\widetilde{F}_{N}^{\varepsilon}\right) \geqslant d-\varepsilon$. В силу произвольности выбора числа $\varepsilon, D_{H}(F) \geqslant D_{H}(\widetilde{F}) \geqslant d=\widehat{D}_{\mathscr{C}}(F)$; по теореме $1.2 \widehat{D}_{\mathscr{C}}(F) \geqslant D_{\mathscr{C}}(F)$. Отсюда и из (1.9) вытекает утверждение теоремы.

Хаусдорфова размерность $D_{H}(\mu)$ конечной борелевской меры $\mu$ на метрическом пространстве $\Lambda$ определяется следующим образом:

$$
D_{H}(\mu)=\inf \left\{D_{H}(Z): Z \subset \Lambda, \mu(Z)=\mu(\Lambda)\right\} .
$$

Следующее предложение является аналогом теоремы Янг [27], в которой рассматривается связь «шаровой» локальной размерности и хаусдорфовой размерности мер в $\mathbf{R}^{n}$.

Следствие 1.1. Пусть $\mu$ - конечная $\rho$-борелевская мера на $\Lambda \subset$ $S^{T}$, и пусть выполнены условия (ССЦ), (КИМ) $u(\mu-\mathrm{P})$. Ecли $d_{\mathscr{C}, \mu}(x)=$ $d \mu$-почти всюду на $\Lambda$, то $D_{H}(\mu)=d$.

Доказате ль с т о. Пусть

$$
G=\left\{x: \lim _{n \rightarrow \infty} \frac{\ln \left[\mu\left(C_{n}(x)\right)\right]}{\ln \left[\beta_{n}(x)\right]}=d\right\} .
$$

Используя теорему 1.6 , получаем $D_{H}(\mu) \leqslant D_{H}(G)=d$. С другой стороны, пусть $\mu(Z)=\mu(\Lambda)$; тогда $\mu(Z \cap G)=\mu(\Lambda)>0$, и, по той же теореме, $D_{H}(Z \cap G)=d ;$ поэтому $D_{H}(\mu) \geqslant \inf \left\{D_{H}(Z \cap G): Z \subset \Lambda, \mu(Z)=\mu(\Lambda)\right\}=$ d. Тем самым следствие доказано.

\section{§2. Масштабные метрики в пространстве последовательностей}

Как и раньше, $S$ - конечное множество; пусть $N$ - число элементов этого множества (мы предполагаем, что $N>1$; не умаляя общности, можно отождествить $S$ с множеством $\{1, \ldots, N\}) ; \mathbf{N}$ и $\mathbf{R}$ означают, соответственно, множества всех натуральных и вещественных чисел; $S^{\mathbf{N}}$ пространство всех $S$-значных последовательностей $x=\left(x_{1}, x_{2}, \ldots\right)$. Для любой последовательности $x \in S^{\mathbf{N}}$ мы рассматриваем цилиндрическое 
множество $C_{n}(x)=\left\{y: y \in \in S^{\mathbf{N}}, y_{1}=x_{1}, \ldots, y_{n}=x_{n}\right\} ; \not{A}$ обозначает $\sigma$-алгебру в $S^{\mathbf{N}}$, порожденную всеми цилиндрическими множествами; $\mathscr{T}$ обозначает топологию, порожденную этими множествами.

Фиксируем целое число $m \geqslant 0$; если $m=0$, зададим функции $\lambda_{n}(s), s \in S$, со значениями в $[0,1)$, которые мы называем функциями масштаба; если $m \geqslant 1$, мы зададим условные функиии масштаба $\lambda_{n}\left(s \mid s_{1}, \ldots, s_{m}\right), n \geqslant m+1$, со значениями в $[0,1)$ (здесь $s, s_{k} \in S$ $(1 \leqslant k \leqslant m))$. Мы будем называть значения функций $\lambda_{n}(s)$ коэффиииентами масштаба, а значения функций $\lambda_{n}\left(s \mid s_{1}, \ldots, s_{m}\right)-$ условными коэффичиентами масштаба в состоянии $s$ при условии, что до этого мы находились в состояниях $s_{1}, \ldots, s_{m} ; m$ называется памятью маситаба; если $m=0$, мы говорим, что у маситаба нет памяти. Для единообразия обозначений мы часто будем писать $\lambda_{n}\left(s \mid s_{1}, \ldots, s_{n}\right)$ вместо $\lambda_{n}(s)$ в случае $m=0$; надеемся, что это не вызовет затруднений.

Выберем также натуральное число $n_{0} \geqslant m$. Если $n_{0}=m=0$, мы обозначим: $a_{n}(x)=\prod_{i=1}^{n} \lambda_{i}\left(x_{i}\right)$ при $n \geqslant 1$; если $n_{0}>0$, то

$$
a_{n}(x)=\prod_{i=n_{0}+1}^{n} \lambda_{i}\left(x_{i} \mid x_{i-m}, \ldots, x_{i-1}\right), \quad n>n_{0} .
$$

При $n_{0}>0$ мы рассмотрим некоторое множество $\tilde{\Lambda}_{0} \subset S^{n_{0}}$ и цилиндрическое множество $\Lambda_{0}=\left\{x: x \in S^{\mathbf{N}},\left(x_{1}, \ldots, x_{n_{0}}\right) \in \widetilde{\Lambda}_{0}\right\}$; обозначим

$$
\begin{aligned}
\Lambda & =\left\{x \in S^{\mathbf{N}}: \lambda_{i}\left(x_{i} \mid x_{i-m}, \ldots, x_{i-1}\right)>0, i>n_{0}\right\} \cup \Lambda_{0}, \\
\Lambda_{n} & =\left\{x \in S^{\mathbf{N}}: a_{n}(x)>0\right\} \cup \Lambda_{0}, \quad n>n_{0} .
\end{aligned}
$$

Ясно, что $\Lambda=\cap_{n=n_{0}+1}^{\infty} \Lambda_{n}$ и $\Lambda_{n+1} \subset \Lambda_{n}, n>n_{0}$. Каждая функция $a_{n}(x)$ постоянна на цилинцрических множествах $C_{n}\left(x^{(i)}\right)$. Поэтому $\Lambda_{n}-$ открыто-замкнутые множества в топологии $\mathscr{T}$. Из сказанного вытекает, что множество $\Lambda$ замкнуто $u \Lambda \in \mathscr{A}$.

Множество $\Lambda$ можно интерпретировать как множество всех «допустимых», или «разрешенных», последовательностей. $B$ дальнейшем мы предполагаем, ито $\Lambda \neq \varnothing$, коль скоро $n_{0}>0 ;$ если же $n_{0}=m=0$, то $\Lambda=\varnothing$.

Обозначим $\widetilde{C}_{n}(x)=C_{n}(x) \cap \Lambda$.

Рассмотрим в $\Lambda$ метрику $\rho$. Обозначим через $B(x, r)$ открытый шар в $\Lambda$ радиуса $r$ с центром в $x \in \Lambda ;|A|$ означает диаметр множества $A \subset \Lambda$, $[A]-\rho$-замыкание этого множества; $A^{\prime}=S^{\mathbf{N}} \backslash A ; \mathscr{T}_{\Lambda}-$ сужение на $\Lambda$ топологии $\mathscr{T}$.

1. Масштабные метрики. Мы будем говорить, что метрика $\rho$ в множестве $\Lambda$ есть маситабная метрика с коэффичиентами масштаба $\lambda_{n}\left(s \mid s_{1}, \ldots, s_{m}\right)$, если существуют положительные постоянные $b$ и $b_{1}$ такие, что при $\beta_{n}(x)=b_{1} a_{n}(x), N_{0}=n_{0}+1$ выполнены условия (ССЦ) и (КИМ) из §1. 
Иными словами, при любом $x \in \Lambda$ и при любом $n>n_{0}$ выполнены следующие условия:

(ССЦ) Скорость сжатия иилиндрических множеств:

$$
\left|C_{n}(x)\right| \leqslant b_{1} a_{n}(x)
$$

(КИМ) Конечность индекса Морана: $L(b)<\infty$, где $L-$ индекс Морана относительно функций $\beta_{n}(x)=b_{1} a_{n}(x)$.

3 а м е ч а н и 2.1. Масштабные метрики, определяемые данным набором функций $\lambda_{n}$, могут быть не эквивалентны.

Пространство $(\Lambda, \rho)$ называется маситабным метрическим пространством. Если при $n>n_{0}$ коэффициенты $\lambda_{n}\left(s \mid s_{1}, \ldots, s_{m}\right)$ не зависят от $n$, мы говорим, что метрика $\rho$ стационарна; в этом случае пространство $(\Lambda, \rho)$ называется стационарным масштабным метрическим пространством.

2. Равномерно разделяющие масштабные метрики. Метрика $\rho$ в $\Lambda$ называется равномерно разделяющей масштабной метрикой, если выполнены условие (ССЦ) и следующее условие:

(РОЦ) Равномерная отделимость иилиндрических множеств: существует положительная постоянная $b_{2}$ такая, что при любых $x \in \Lambda$, $n \in \mathbf{N}$

$$
\text { если } y \notin \widetilde{C}_{n}(x), \text { то } \rho(x, y)>b_{2} a_{n}(x) \text {. }
$$

3 а м е ч а н и е 2.2. В отличие от условия (КИМ), условия (ССЦ) и (РОЦ) можно сформулировать в эквивалентных наглядных «геометрических» терминах: при любом $x \in \Lambda$ и при любом $n>n_{0}$

$$
\text { если } r>b_{1} a_{n}(x), \text { то } \widetilde{C}_{n}(x) \subset B(x, r) ;
$$

если $r \leqslant b_{2} a_{n}(x)$, то при любом $z \in \widetilde{C}_{n}(x)$ шар $B(z, r) \subset \widetilde{C}_{n}(x)$.

Таким образом, если условие (РОЩ) выполнено, то выполнены условия (ЖЦ) (см. §1) и (КИМ), причем $L\left(b_{2} / b_{1}\right)=1$.

П р и м е 2.1 . Рассмотрим числа $m$ и $n_{0}$, функции $a_{n}(x)$, определенные выше, и положительное число $c$; запись $x \sim^{n} y$ означает: $x_{1}=y_{1}, \ldots, x_{n}=y_{n}, x_{n+1} \neq y_{n+1}$. Легко убедиться, что

$$
\rho(x, y)= \begin{cases}c, & \text { если } x_{n} \neq y_{n} \text { при некотором } n \leqslant n_{0} \\ c a_{n-n_{0}}(x)=c a_{n-n_{0}}(y), & \text { если } x \sim y \text { при некотором } n>n_{0} \\ 0, & \text { если } x=y\end{cases}
$$

является равномерно разделяющей масштабной метрикой в $\Lambda$ (при этом мы можем положить $\left.b_{1}=b_{2}=c\right)$. Замечание 2.2 показывает, что в этом случае $\widetilde{C}_{n}(x)=B(x, r)$, если $c a_{n}(x)<r<c a_{n-1}(x), x \in \Lambda$. 
П р и м е р 2.2. Пусть $m=0, N<\infty, \lambda(s) \equiv \lambda$ и пусть $\phi$-метрика в $S$; обозначим: $\alpha=\min _{x, y \in S, x \neq y} \phi(x, y) ; \beta=\max _{x, y \in S} \phi(x, y)$. Если $x=\left(x_{1}, x_{2}, \ldots\right)$ и $y=\left(y_{1}, y_{2}, \ldots\right)$, мы положим

$$
\rho(x, y)=\sum_{n=1}^{\infty} \lambda^{n} \phi\left(x_{n}, y_{n}\right)
$$

Легко убедиться, что $\rho$ - равномерно разделяюшая масштабная метрика в пространстве $S^{\mathbf{N}}$, причем $b_{1}=\beta \lambda /(1-\lambda), b_{2}=\alpha$.

Широкий класс масштабных метрик в $\Lambda$ индуџируется фракталами в обших метрических пространствах (см. ниже §5).

3. Маситабиые метрики со свойством (СР). Пусть $\Lambda \subset S^{\mathbf{N}}$ и $\rho-$ масштабная метрика в $\Lambda$ с коэффициентами $\lambda_{k}(\cdot \mid \cdot)$. Обозначим:

$$
\begin{gathered}
g=\inf \left\{\lambda_{k}\left(s \mid s_{1}, \ldots, s_{m}\right) ; s_{1}, \ldots, s_{m}, s \in S\right. \\
\left.\lambda_{k}\left(s \mid s_{2} \ldots, s_{m}\right)>0, k \in \mathbf{N}\right\} .
\end{gathered}
$$

Условие (СР) выполнено, если $g>0$, причем можно положить $\gamma=g$.

4. Усреднимые масштабные метрики. Обсудим, наконец, условие $(\mu-\mathrm{P})$ кз $\S 1$.

Будем говорить, что масштабная метрика $\rho$ усреднима относительно меры $\mu$ (или просто $\mu$-усреднима), если выполнено следующее условие:

(У) для $\mu$-почти всех $x \in \Lambda$ существует конечный положительный предел

$$
\lim _{n \rightarrow \infty} \frac{1}{n} \ln a_{n}(x)=\lim _{n \rightarrow \infty} \frac{1}{n} \sum_{i=m+1}^{n} \ln \lambda_{i}\left(x_{i} \mid x_{i-m}, \ldots, x_{i-1}\right) \stackrel{\text { def }}{=}-\bar{h}_{\lambda}(x)
$$

(сходное условие было введено в работе [25]). Легко убедиться в том, что всякая усреднимая метрика удовлетворяет условию $(\mu-\mathrm{P})$. Как показывают приведенные ниже примеры, это понятие тесно связано с эргодической теоремой, законом болыших чисел и теоремами о средних значениях.

П р и м е р 2.3. Пусть $\rho$ - стационарная масштабная метрика и $\mu-$ трансляционно инвариантная конечная мера на $\not \mathcal{~}$. Обозначим: $\tilde{\mu}-$ трансляционно инвариантная мера на $S^{\mathbf{Z}}$, совпадающая с $\mu$ на $S^{\mathbf{N}}$;

$$
\begin{aligned}
h_{\mu} & =-\int_{\Lambda}\left[\ln \mu\left\{x_{1} \mid \ldots, x_{-1}, x_{0}\right\}\right] \tilde{\mu}(d x) ; \\
h_{\lambda, \mu} & =-\int_{\Lambda}\left[\ln \lambda\left(x_{m+1} \mid x_{1}, \ldots, x_{m}\right)\right] \mu(d x) .
\end{aligned}
$$

Условие (У) выполнено в силу эргодической теоремы Биркгофа; поскольку коэффициенты масштаба $\lambda\left(x_{m+1} \mid x_{1}, \ldots, x_{m}\right)<1$, то $\mu$-п.в. $\bar{h}_{\lambda}(x)>0$. 
Таким образом, любая стачионарная масштабная метрика усреднима относительно всякой транслячионно инвариантной меры $\mu$. Очевидно, если мера $\mu$ эргодична, то $\bar{h}_{\lambda}(x) \equiv h_{\lambda, \mu}$.

П р и м е р 2.4. Если для любого $x \in \Lambda$ последовательность $\left\{-\ln \lambda_{n}\left(x_{n} \mid x_{n-m}, \ldots, x_{n-1}\right)\right\}$ почти периодична по Безиковичу и ее среднее значение $\bar{h}_{\lambda}(x)>0$, то метрика $\rho$ усреднима относительно любой конечной меры.

П р и м е р 2.5. Пусть $m=0$ и $\mathbf{P}$ - вероятностная мера на $\mathscr{A}$, относительно которой «координатные» случайные величины $X_{1}, X_{2}, \ldots$ независимы; кроме того, пусть существует предел $\lim _{n \rightarrow \infty} n^{-1} \sum_{k=1}^{n} \mathbf{E}\left[\ln \lambda_{k}\left(X_{k}\right)\right]=-\bar{h}_{\lambda}>0$ и $\sum_{k=1}^{\infty} \mathbf{D}\left[\ln \lambda_{k}\left(X_{k}\right)\right] / k^{2}<\infty$. Согласно усиленному закону больших чисел Колмогорова, метрика $\rho$ P-усреднима.

Еще один важный класс усреднимых нестационарных метрик случайные масштабные метрики - будет рассмотрен в $\$ 4$.

5. Некоторые свойства масштабных метрических пространств. Для удобства ссылок мы переформулируем основной результат §1теорему 1.6.

Теорема 2.1. Пусть $F \in \mathscr{A}, \mu-$ конечная мера на $\mathscr{A}, \mu(F)=$ $\mu\left(S^{\mathbf{N}}\right)$, и пусть $\rho$ есть $\mu$-усреднимая маситабная метрика в $\Lambda$. Предположим, что всюду на множестве $F \in \mathscr{A}$ определена размерность $d_{\mathscr{C}, \mu}(x) \equiv d$. Tогда $D_{H}(F)=D_{\mathscr{C}}(F)=\widehat{D}_{\mathscr{C}}(F)=d$.

Мы рассмотрим ниже условия сушествования локальных размерностей.

Обозначим через $\tau$ преобразование сдвига в $S^{\mathbf{N}}$, определенное, как обычно: если $x=\left(x_{1}, x_{2}, \ldots\right)$, то $(\tau x)_{i}=x_{i+1}$. Под трансляционной инвариантностью или эргодичностью меры мы понимаем соответствующее ее свойство относительно некоторой полугруппы сдвигов $\tau^{k}, k \geqslant k_{0}$.

Теорема 2.2. Пусть $\mu$ - эргодическая транслячионно инвариантная конечная мера на $\mathscr{A}$, причем $\mu\left(S^{\mathbf{N}}\right)=\mu(\Lambda)$, и $\rho$ есть $\mu$-усреднимая масштабная метрика. Тогда $\mu$-п.в. определена локальная размерность $d_{\mathscr{C}, \mu} u$

$$
d_{\mathscr{C}, \mu}(x)=\frac{h_{\mu}}{\bar{h}_{\lambda}(x)}
$$

$B$ частности, если $\rho$ стачионарна, то $d_{\zeta_{, \mu}}(x)=h_{\mu} / h_{\lambda, \mu}$.

Д о к а з а т е л в с т в о. Из «обрашенного назад» варианта теоремы ШІеннона-Макмиллана-Бреймана вытекает, что $\mu$-п.в.

$$
\lim _{n \rightarrow \infty} \frac{1}{n} \ln \mu\left(C_{n}(x)\right)=-h_{\mu} .
$$

Остается воспользоваться соотношениями (1.4) и (2.4). 
Следуюшие предложения непосредственно вытекают из теорем 2.1, 2.2 и следствия 1.1.

Следствие 2.1. Если выполнены условия теоремы 2.1, причем мера $\mu$ трансляционно инвариантна и эргодична, то $D_{H}(F)=D_{\mathscr{C}}(F)=$ $\widehat{D}_{\mathscr{C}}(F)=h_{\mu} / h_{\lambda, \mu}$.

Следствие 2.2. Пусть $\mu-$ эргодическая транслячионно инвариантная мера в пространстве $S^{\mathbf{N}}$, оснащенном стачионарной масштабной метрикой с функциями маситаба $\lambda(\cdot \mid \cdot) ;$ mогда $D_{H}(\mu)=h_{\mu} / h_{\lambda, \mu}$.

3 а м е ч а н и е 2.3. Теорема 1.4 и теорема 2.10 в [24] показывают, что определенная в [24] насыщенная корреляционная размерность сдвигов в стационарном масштабном метрическом пространстве $\Lambda$, соответствующая эргодической трансляционно инвариантной мере $\mu$, совпадает c $h_{\mu} / h_{\lambda, \mu}$.

3 а м е ч а н и е 2.4. Отметим, что при фиксированных коэффициентах масштаба локальные размерности $d_{\mathscr{B}, \mu}$ и $d_{\mathscr{C}, \mu}$ не зависят от выбора масштабной метрики $\rho$.

П р и м е р 2.6. (Масштабные метрики без памяти.) Пусть $m=0$. Рассмотрим произвольную масштабную метрику $\rho$ с коэффициентами масштаба $\lambda(b), b \in S$. Обозначим через $\alpha_{0}$ (единственное) решение уравнения $\sum_{\alpha \in S} \lambda(b)^{\alpha}=1$. Рассмотрим случайную последовательность Бернулли с маргинальным распределением $p(b)=\lambda(b)^{\alpha_{0}}, b \in S$; меру $P$ в $S^{\mathbf{N}}$, соответствуюшую этой последовательности, мы называем маситабной мерой Бернулли. Метрика $\rho P$-усреднима (см. пример 2.3). Ясно, что $P\left(C_{n}(x)\right)=\left[a_{n}(x)\right]^{\alpha_{0}}$. Таким образом, всюду на $S^{\mathbf{N}}$ суптествует $d_{\mathscr{C}, P}=\alpha_{0}$. По теореме $2.1 \alpha_{0}=D_{H}\left(S^{\mathbf{N}}\right)$. В силу теоремы $1.4, d_{\mathscr{B}, P}=d_{\mathscr{C}, P}$. Мы имеем: $h_{P}=\alpha_{0} \sum_{b \in S} \lambda(b)^{\alpha_{0}} \ln (1 / \lambda(b))$; $h_{\lambda, P}=\sum_{b \in S} \lambda(b)^{\alpha_{0}} \ln (1 / \lambda(b))$, и, таким образом, $D_{H}\left(S^{\mathbf{N}}\right)=d_{\mathscr{B}, P}=$ $d_{\mathscr{C}, P}=\alpha_{0}=h_{P} / h_{\lambda, P}$.

В § 3-5 результат, полученный в примере 2.6, обобщен в различных направлениях; примененный здесь метод нахождения хаусдорфовой размерности, основанный на построении адекватной «масштабной» меры и вычислении ее локальной размерности, использован и в дальнейшем.

\section{§ 3. Масштабные меры и хаусдорфова размерность в $S^{\mathbf{N}}$}

Мы используем здесь обозначения, введенные в §2. Предположим, что $\rho$ - стационарная масштабная метрика с коэффициентами $\lambda\left(s_{m+1} \mid\right.$ $\left.s_{1}, \ldots, s_{m}\right)$. Пусть $m>0$; обозначим: $\Phi=\mathbf{R}^{S^{m}}$ (пространство всех $N^{m}$-мерных векторов $\left.\left\{y\left(s_{1}, \ldots, s_{m}\right), s_{1}, \ldots, s_{m} \in S\right\}\right)$. При любом $\alpha>0$ мы рассматриваем в $\Phi$ оператор $V_{\alpha}: y \mapsto V_{\alpha} y=z$, где

$$
z\left(x_{1}, \ldots, x_{m}\right)=\sum_{x_{m+1} \in S} \lambda^{\alpha}\left(x_{m+1} \mid x_{1}, \ldots, x_{m}\right) y\left(x_{2}, \ldots, x_{m}, x_{m+1}\right) .
$$


Очевидно, что $V_{\alpha}$ - неотрицательный линейный оператор. $B$ дальнейием мы предполагаем, что операторы $V_{\alpha}$ неразложимы, т.е. для любых $\left(d_{1}, \ldots, d_{m}\right),\left(e_{1}, \ldots, e_{m}\right) \in S^{m}$ мы имеем: $\lambda\left(t_{i+1} \mid t_{i-m+1}, \ldots, t_{i}\right)>0$, $m \leqslant i \leqslant n-1$, при некоторых $t_{1}, \ldots, t_{n}$ (здесь $t_{1}=d_{1}, \ldots, t_{m}=d_{m}$, $\left.t_{n-m+1}=e_{1}, \ldots, t_{n}=e_{m}\right)$.

Напомним, что спектральный радиус $r(\alpha)$ оператора $V_{\alpha}$ - это наибольший модуль его собственных значений; по теореме ПерронаФробениуса (см., например, гл. $2, \S 2$ в [1]), $r(\alpha)$ является собственным значением оператора $V_{\alpha}$. Рассмотрим обобщенное уравнение Морана:

$$
r(\alpha)=1 \text {. }
$$

Функция $r(\cdot)$ - непрерывная убывающая функция, причем $r(0) \geqslant 1$, $\lim _{\alpha \rightarrow \infty} r(\alpha)=0$; поэтому существует единственная точка $\alpha_{0}$ такая, что $r\left(\alpha_{0}\right)=1$ (единственность решения уравнения (3.2) вытекает также из теоремы 3.1 , приведенной ниже).

Согласно теореме Перрона-Фробениуса, сушествует положительный инвариантный вектор $\left\{w\left(x_{1}, \ldots, x_{m}\right), x_{1}, \ldots, x_{m} \in S\right\}$ oператора $V_{\alpha_{0}}$. Рассмотрим функцию

$$
p\left(x_{m+1} \mid x_{1}, \ldots, x_{m}\right)=\frac{\lambda^{\alpha_{0}}\left(x_{m+1} \mid x_{1}, \ldots, x_{m}\right) w\left(x_{2}, \ldots, x_{m+1}\right)}{w\left(x_{1}, \ldots, x_{m}\right)}
$$

Легко убедиться, что

$$
\sum_{x_{m+1}} p\left(x_{m+1} \mid x_{1}, \ldots, x_{m}\right)=\frac{w\left(x_{1}, \ldots, x_{m}\right)}{w\left(x_{1}, \ldots, x_{m}\right)}=1
$$

Поэтому $p\left(x_{m+1} \mid x_{1}, \ldots, x_{m}\right)$ является вероятностью перехода порядка $m$.

Мы полагаем здесь $\tilde{\Lambda}_{0}=S^{m}$ при $n_{0}=m$ и $\tilde{\Lambda}_{0}=\left\{\left(x_{1}, \ldots, x_{n_{0}}\right): \lambda\left(x_{i} \mid\right.\right.$ $\left.\left.x_{i-m}, \ldots, x_{i-1}\right)>0, m<i \leqslant n_{0}\right\}$ при $n_{0}>m$. Согласно теореме Перрона-Фробениуса, оператор $V_{\alpha_{0}}^{*}$ обладает положительным инвариантным вектором $v\left(x_{1}, \ldots, x_{m}\right)$; мы нормируем его условием $\sum_{x_{i} \in S} w\left(x_{1}, \ldots, x_{m}\right) v\left(x_{1}, \ldots, x_{m}\right)=1$. Обозначим: $p_{0}\left(x_{1}, \ldots, x_{n_{0}}\right)=$ $w\left(x_{n_{0}-m+1}, \ldots, x_{n_{0}}\right) v\left(x_{n_{0}-m+1}, \ldots, x_{n_{0}}\right)$ при $n_{0}=m$ и $p_{0}\left(x_{1}, \ldots, x_{n_{0}}\right)=$ $w\left(x_{n_{0}-m+1}, \ldots, x_{n_{0}}\right) v\left(x_{n_{0}-m+1}, \ldots, x_{n_{0}}\right) \prod_{i=m+1}^{n_{0}} p\left(x_{i} \mid x_{i-m}, \ldots, x_{i-1}\right)$ при $n_{0}>m$.

Масштабной мерой в $S^{\mathbf{N}}$, определяемой функцией масштаба $\lambda$, мы называем марковскую (порядка $m$ ) меру $\mu$ в $S^{\mathbf{N}}$ с вероятностями перехода $p\left(x_{m+1} \mid x_{1}, \ldots, x_{m}\right)$ и начальным распределением $p_{0}$.

Таким образом, если $x \in \Lambda$ и $n>n_{0}$, то

$$
\begin{aligned}
\mu\left(C_{n}(x)\right) & =\prod_{i=n_{0}+1}^{n} p\left(x_{i} \mid x_{i-m}, \ldots, x_{i-1}\right) p_{0}\left(x_{1}, \ldots, x_{n_{0}}\right) \\
& =\left(a_{n-n_{0}}(x)\right)^{\alpha_{0}} \frac{w\left(x_{n-m+1}, \ldots, x_{n}\right)}{w\left(x_{n_{0}-m+1}, \ldots, x_{n_{0}}\right)} p_{0}\left(x_{1}, \ldots, x_{n_{0}}\right) .
\end{aligned}
$$


Хорошо известно, что мера $\mu$ трансляционно инвариантна и эргодична; следовательно, метрика $\rho$ является $\mu$-усреднимой.

Ясно, что $\psi_{0} \stackrel{\text { def }}{=} \inf \left\{p_{0}\left(x_{1}, \ldots, x_{n_{0}}\right):\left(x_{1}, \ldots, x_{n_{0}}\right) \in \tilde{\Lambda}_{0}\right\}>0$.

Легко убедиться, что $\mu\left(\Lambda_{n}\right)=\mu\left(\tilde{\Lambda}_{0}\right)>0$ при любом $n>n_{0}$, и, поскольку $\Lambda_{n} \downarrow \Lambda$, мы имеем: $\mu(\Lambda)=\mu\left(\widetilde{\Lambda}_{0}\right)>0$. Далее, очевидно, что при $n>n_{0}$

$$
\psi_{0} m_{0}^{-1}\left(a_{n}(x)\right)^{\alpha_{0}} \leqslant \mu\left(C_{n}(x)\right) \leqslant m_{0}\left(a_{n}(x)\right)^{\alpha_{0}},
$$

где $m_{0}=\max \left\{w\left(s_{1}, \ldots, s_{m}\right)\right\} / \min \left\{w\left(s_{1}, \ldots, s_{m}\right)\right\}$.

Если $m=0$, то $\mu$ - масштабная мера Бернулли, определенная в примере 2.6. В этом случае $\mu\left(C_{n}(x)\right)=\left(a_{n}(x)\right)^{\alpha_{0}}$.

Поскольку $a_{n}(x) \rightarrow 0$, то для любой последовательности $x \in \Lambda$ существует локальная размерность

$$
d_{\mathscr{C}, \mu}(x)=\lim _{n \rightarrow \infty} \frac{\ln \mu\left(C_{n}(x)\right)}{\ln \left[a_{n}(x)\right]}=\alpha_{0} .
$$

Из теоремы 2.1 при $F=\Lambda$ и следствия 2.1 вытекает следующее утверждение.

Tеорема 3.1. Хаусдорфова размерность $D_{H}(\Lambda)=\alpha_{0}=h_{\mu} / h_{\lambda, \mu}$. Напомним, что $\Lambda=S^{\mathbf{N}}$, если все коэффициенты масштаба положительны.

П р и м е р 3.1. Пусть $S=\{1, \ldots, N\}$. Рассмотрим в $S^{\mathbf{N}}$ масштаб без памяти с коэффициентами $\lambda_{1}, \ldots, \lambda_{N}$ :

$$
V_{\alpha}=\left(\begin{array}{cccc}
\lambda_{1}^{\alpha} & \lambda_{2}^{\alpha} & \ldots & \lambda_{N}^{\alpha} \\
\vdots & \vdots & \ddots & \vdots \\
\lambda_{1}^{\alpha} & \lambda_{2}^{\alpha} & \ldots & \lambda_{N}^{\alpha}
\end{array}\right)
$$

В этом случае $r(\alpha)=\sum_{i} \lambda_{i}^{\alpha}$, и уравнение (3.2) сводится к уравнению Морана $\sum_{i} \lambda_{i}^{\alpha}=1$ (см. [22]), причем $\left(w_{1}, \ldots, w_{N}\right)=(1, \ldots, 1)$; масштабная мера представляет собой меру Бернулли с $p(i)=\lambda_{i}^{\alpha_{0}} ;$ таким образом, $D_{H}\left(S^{\mathbf{N}}\right)=\alpha_{0}$. Если $\lambda_{i}=\lambda, i=1, \ldots, N$, то $D_{H}\left(S^{\mathbf{N}}\right)=\ln N / \ln (1 / \lambda)$. Если масштаб имеет память 1 , то

$$
V_{\alpha}=\left(\begin{array}{cccc}
\lambda^{\alpha}(1 \mid 1) & \lambda^{\alpha}(2 \mid 1) & \ldots & \lambda^{\alpha}(N \mid 1) \\
\vdots & \vdots & \ddots & \vdots \\
\lambda^{\alpha}(1 \mid N) & \lambda^{\alpha}(2 \mid N) & \ldots & \lambda^{\alpha}(N \mid N)
\end{array}\right) .
$$

Если при некотором $\alpha_{0}$ матрица $V_{\alpha_{0}}-$ стохастическая, то $r\left(V_{\alpha_{0}}\right)=1$, $\left(w_{1}, \ldots, w_{N}\right)=(1, \ldots, 1)$ и $D_{H}\left(S^{\mathbf{N}}\right)=\alpha_{0}$.

Следующее утверждение является уточнением предыдущей теоремы для равномерно разделяющих метрик. 


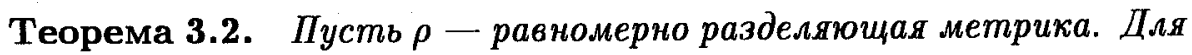
любого борелевского множества $F \subset \Lambda$ и для любой масштабной меpbl $\mu$

$$
\begin{aligned}
m_{0}^{-1}\left(2 b_{2}\right)^{\alpha_{0}} \mu(F) & \leqslant H_{\mathscr{C}}^{\alpha_{0}}(F) \leqslant \psi_{0}^{-1} m_{0}\left(b_{1}\right)^{\alpha_{0}} \mu(F), \\
b_{1}^{-\alpha_{0}} b_{2}^{2 \alpha_{0}} g^{\alpha_{0}} m_{0}^{-1} \mu(F) & \leqslant H^{\alpha_{0}}(F) \leqslant \psi_{0}^{-1} m_{0}\left(b_{1}\right)^{\alpha_{0}} \mu(F),
\end{aligned}
$$

где постоянная $g$ определена соотношением (2.3). Следовательно, хаусдорбова размерность точна.

Д о к а 3 а т е л ь с т в о. Из свойств равномерно разделяюших метрик следует, что

$$
\left(2 b_{2}\right)^{\alpha_{0}} \sum_{i}\left(a_{n_{i}}\left(x^{(i)}\right)\right)^{\alpha_{0}} \leqslant \sum_{i}\left|\widetilde{C}_{n_{i}}\left(x^{(i)}\right)\right|^{\alpha_{0}} \leqslant\left(b_{1}\right)^{\alpha_{0}} \sum_{i}\left(a_{n_{i}}\left(x^{(i)}\right)\right)^{\alpha_{0}} .
$$

Из этих неравенств и соотношения (3.3) вытекает, что

$$
H_{n, \mathscr{C}}^{\alpha_{0}}(F) \leqslant \psi_{0}^{-1} m_{0}\left(b_{1}\right)^{\alpha_{0}} \inf \left\{\sum_{i} \mu\left(C_{n_{\mathfrak{i}}}\left(x^{(i)}\right)\right)\right\},
$$

где нижняя грань берется по всем покрытиям $\left\{C_{n_{i}}\left(x^{(i)}\right)\right\}$ множества $F$ щилиндрическими множествами с номерами $n_{i} \geqslant n$. По теореме об аппроксимации мер, $\inf \sum_{i} \mu\left(C_{n_{i}}\left(x^{(i)}\right)\right)=\mu(F)$, и, следовательно, $\widehat{H}_{n, \mathscr{C}}^{\alpha_{0}}(F) \leqslant \psi_{0}^{-1} m_{0}\left(b_{1}\right)^{\alpha_{0}} \mu(F)$. Кроме того, $\sum_{i}\left(a_{n_{i}}\left(x^{(i)}\right)\right)^{\alpha_{0}} \geqslant$ $m_{0}^{-1} \sum_{i} \mu\left(C_{n_{i}}\left(x^{(i)}\right)\right) \geqslant m_{0}^{-1} \mu\left(\cup_{i} C_{n_{i}}\left(x^{(i)}\right)\right) \geqslant m_{0}^{-1} \mu(F)$, и поэтому $H_{n, \mathscr{C}}^{\alpha_{0}}(F) \geqslant m_{0}^{-1}\left(2 b_{2}\right)^{\alpha_{0}} \mu(F)$. Остается сослаться на теорему 1.3 и теорему 1.2 .

3 а м е ч а н и е 3.1.- Из теоремы 3.2 и соотношения (3.4) вытекает, что локальная размерность хаусдорфовой меры $H^{\alpha_{0}}$ тождественно равна $\alpha_{0}$. Таким образом, согласно следствию 1.1 , хаусдорбова размерность меры $H^{\alpha_{0}}$ равна хаусдорфовой размерности множества $\Lambda$.

3 а м е ч а н и е 3.2 . Теоремы 2.2 и 1.5 показывают, что для любой масштабной метрики с коэффициентами $\lambda$ и для любой эргодической транслящионно инвариантной меры $\mu$ сушествует множество $\Gamma \subset S^{\mathbf{N}}$ такое, что $D_{H}(\Gamma)=h_{\mu} / h_{\lambda, \mu}$, и поэтому $\alpha_{0} \geqslant h_{\mu} / h_{\lambda, \mu}$ для любой такой меры $\mu$; равенство достигается, например, если $\mu$ - масштабная мера с коэффищиентами $\lambda$.

\section{$\S 4$. Хаусдорфова размерность в $S^{\mathrm{N}}$ относительно случайной масштабной метрики}

Наряду с пространством $S^{\mathbf{N}}$ мы рассмотрим пространство $(\Omega, \mathscr{F})-$ произведение счетного множества копий измеримого пространства $\left(\Omega_{0}, \mathscr{F}_{0}\right)$. Обозначим элементы пространства $\Omega$ через $\omega=\left(\omega_{1}, \omega_{2}, \ldots\right)$, где $\omega_{i} \in \Omega_{0}$. Пусть $Q$ - эргодическая трансляционно инвариантная вероятностная мера на $\mathscr{F}$. 
Обозначим через $\tau$ и $\sigma$ сдвиги влево в пространствах $S^{\mathbf{N}}$ и $\Omega$ соответственно.

Рассмотрим случайные козффичиенты маситаба без памяти, т.е. функции $\lambda\left(s ; \omega_{0}\right), s \in S, \omega_{0} \in \Omega_{0}$, со значениями в $[0,1)$; предположим, что при каждом выборе состояния $s$ эти коэффициенты являются измеримыми функциями от $\omega_{0}$ и что $\sum_{s \in S} \lambda\left(s ; \omega_{0}\right)>0$ при любом $\omega_{0} \in \Omega_{0}$. Обозначим $a_{n}(x ; \omega)=a_{n}\left(x_{1}, \ldots, x_{n} ; \omega\right)=\prod_{i=1}^{n} \lambda\left(x_{i} ; \omega_{i}\right) ; \Lambda_{\omega}=$ $\left\{x \in S^{\mathbf{N}}: \lambda\left(x_{i} ; \omega_{i}\right)>0, i \in \mathbf{N}\right\}$.

Мы будем говорить, что семейство функщий $\left\{\rho_{\omega}(x, y), \omega \in \Omega\right\}$ есть случайная масштабная метриха с коэффичиентами маситаба $\lambda\left(s ; \omega_{0}\right)$, если для $Q$-почти каждого $\omega \in \Omega$ функция $\rho_{\omega}(x, y)$ является масштабной метрикой в $\Lambda_{\omega}$ с коэффициентами масштаба $\tilde{\lambda}_{k}(s)=\lambda\left(s ; \omega_{k}\right)$.

Если $\Omega_{0}$ состоит из одной точки, это понятие сводится к понятию стационарной масштабной метрики без памяти (см. §2).

Вычислим хаусдорфову размерность множества $\Lambda_{\omega}$ относительно случайной масштабной метрики $\rho_{\omega}$. При любом $\alpha>0$ и любом $\omega \in \Omega$ мы определим значения функции множеств $\mu_{\omega}^{(\alpha)}$ на цилиндрических множествах следующим образом: $\mu_{\omega}^{(\alpha)}\left(C_{n}(x)\right)=\left[Z_{n}(\omega)\right]^{-1} \prod_{i=1}^{n} \lambda^{\alpha}\left(x_{i} ; \omega_{i}\right)$, $n \geqslant 1$, где $Z_{n}(\omega)=\prod_{i=1}^{n}\left[\sum_{x_{i} \in S} \lambda^{\alpha}\left(x_{i} ; \omega_{i}\right)\right]$.

Легко видеть, что при любом $\omega$ эти соотношения определяют вероятностную продакт-меру с маргинальными распределениями $p_{\omega, i}^{(\alpha)}\left(x_{i}\right)=$ $\lambda^{\alpha}\left(x_{i} ; \omega_{i}\right) / \sum_{x_{i} \in S} \lambda^{\alpha}\left(x_{i} ; \omega_{i}\right) ;$ мы называем ее случайной маситабной меpoü.

Заметим, что $\mu_{\omega}^{(\alpha)}\left(C_{n}(x)\right)=0$, если $a_{n}(x ; \omega)=0$. Отсюда легко вывести следующее утверждение.

Лемма 4.1. $C Q$-вероятностью $1 \mu_{\omega}^{(\alpha)}\left(\Lambda_{\omega}\right)=1$.

Рассмотрим вероятностную меру $R^{(\alpha)}$ на $\mathscr{A} \times \mathscr{F}$, заданную соотношением $R^{(\alpha)}(C)=\int_{C} \mu_{\omega}^{(\alpha)}(d x) Q(d \omega), C \in \mathscr{A} \times \mathscr{F}$.

Лемма 4.2. Мера $R^{(\alpha)}$ инвариантна относительно сдвига $\tau \times \sigma$.

Доказ а т л в с т о. Поскольку

$$
\tau^{-1}\left(C_{n}\left(x_{1}, x_{2}, \ldots\right)\right)=\cup_{x_{0} \in S} C_{n+1}\left(x_{0}, x_{1}, x_{2}, \ldots\right),
$$

мы имеем:

$$
\begin{aligned}
\mu_{\omega}^{(\alpha)}\left(\tau^{-1}\left[C_{n}(x)\right]\right) & =\sum_{x_{0} \in S} \frac{\prod_{i=1}^{n+1} \lambda^{\alpha}\left(x_{i-1} ; \omega_{i}\right)}{\prod_{i=1}^{n+1}\left[\sum_{x_{i-1} \in S} \lambda^{\alpha}\left(x_{i-1} ; \omega_{i}\right)\right]} \\
& =\frac{\prod_{i=2}^{n+1} \lambda^{\alpha}\left(x_{i-1} ; \omega_{i}\right)}{\prod_{i=2}^{n+1}\left[\sum_{x_{i-1} \in S} \lambda^{\alpha}\left(x_{i-1} ; \omega_{i}\right)\right]}=\frac{\prod_{i=1}^{n} \lambda^{\alpha}\left(x_{i} ; \omega_{i+1}\right)}{\prod_{i=1}^{n}\left[\sum_{x_{i} \in S} \lambda^{\alpha}\left(x_{i} ; \omega_{i+1}\right)\right]} \\
& =\mu_{\sigma(\omega)}^{(\alpha)}\left(C_{n}(x)\right) .
\end{aligned}
$$

Отсюда следует, что при любых $\omega \in \Omega$ и $A \in \mathscr{A}$ имеем: $\mu_{\omega}^{(\alpha)}\left(\tau^{-1} A\right)=$ 
$\mu_{\sigma(\omega)}^{(\alpha)}(A)$. Поэтому при любых $A \in \mathscr{A}$ и $B \in \mathscr{F}: R^{(\alpha)}\left(\tau^{-1} A \times \sigma^{-1} B\right)=$ $\int_{\sigma^{-1} B} \mu_{\omega}^{(\alpha)}\left(\tau^{-1} A\right) Q(d \omega)=\int_{\sigma^{-1} B} \mu_{\sigma(\omega)}^{(\alpha)}(A) Q(d \omega)=R^{(\alpha)}(A \times B)$. Отскода вытекает наше утверждение.

Обозначим: $\ln ^{-} x=\ln x$, если $0<x<1$, и $\ln ^{-} x=0$, если $x=0$; $h_{\lambda, Q}(s)=-\mathbf{E}\left[\ln ^{-} \lambda\left(s ; \omega_{1}\right)\right], s \in S . B$ дальнейшем мы предполагаем, что выполнено следующее условие:

$$
h_{\lambda, Q}(s)<\infty, \quad s \in S
$$

Лемма 4.3. $C Q$-вероятностью 1 метрика $\rho_{\omega}$ является $\mu_{\omega}^{(\alpha)}$ усреднимой.

Д о к а з а т е л ь с т в о. Из эргодической теоремы Биркгофа вытекает, что с $R^{(\alpha)}$-вероятностью 1 существует конечный предел

$$
\lim _{n \rightarrow \infty} \frac{1}{n} \sum_{i=1}^{n} \ln \lambda\left(x_{i} ; \omega_{i}\right)=-\bar{h}_{\lambda}(x, \omega) .
$$

Поскольку все коэффициенты масштаба $\lambda\left(x_{i} ; \omega_{i}\right)<1$, то с $Q$-вероятностью $1 \bar{h}_{\lambda}(x, \omega)>0$ для $\mu_{\omega}^{(\alpha)}$-почти всех $x \in S^{\mathbf{N}}$. Следовательно, метрика $\rho_{\omega}$ является $\mu_{\omega}^{(\alpha)}$-усреднимой.

Лемма 4.4. 1 . $C$ Q-вероятностью 1 существует предел $\lim _{n \rightarrow \infty} n^{-1} \ln Z_{n}(\omega) \stackrel{\text { def }}{=} P(\alpha)$.

2. $P(\alpha)=\mathbf{E}\left[\ln \sum_{s \in S} \lambda^{\alpha}\left(s ; \omega_{0}\right)\right]$.

Д ок а 3 а т ель с т в о. При любых $s_{0} \in S, \omega_{0} \in \Omega_{0}$ имеем: $\alpha \ln \left[\lambda\left(s_{0} ; \omega_{0}\right)\right] \leqslant \ln \left[\sum_{s \in S} \lambda^{\alpha}\left(s ; \omega_{0}\right)\right] \leqslant \sum_{s \in S} \lambda^{\alpha}\left(s ; \omega_{0}\right) \leqslant N ;$ в силу (4.1), $\mathrm{E}\left[\left|\ln \left[\sum_{s \in S} \lambda^{\alpha}\left(s ; \omega_{0}\right)\right]\right|\right]<\infty$. Поэтому, в силу эргодической теоремы Биркгофа, с $Q$-вероятностью 1 существует предел $\lim _{n \rightarrow \infty} n^{-1} \ln Z_{n}(\omega)=$ $\lim _{n \rightarrow \infty} n^{-1} \sum_{i=1}^{n} \ln \left[\sum_{s \in S} \lambda^{\alpha}\left(s ; \omega_{i}\right)\right]$; утверждение 2 справедливо в силу эргодичности меры $Q$.

3 а м е ч а н и е 4.1. При любом $\omega \in \Omega$ меру $\mu_{\omega}^{(\alpha)}$ можно трактовать как гиббсовское случайное поле на $\mathbf{N}$, определяемое одночастичным потенциалом $U_{\omega}\left(x_{i}\right)=-\ln \lambda\left(x_{i} ; \omega_{i}\right)$ и температурой $\alpha^{-1} ;$ величина $P(\alpha)$ представляет собой цавление, соответствуюшее этому полю.

Лемма 4.5. Уравнение

$$
P(\alpha)=0
$$

имеет одно и только одно решение.

Д ок а з т е льство. Из утверждения 2 леммы 4.4 вытекает, что $P(\cdot)$ - непрерывная убывающая функция; остается заметить, что $P(0)=\ln N>0, \lim _{\alpha \rightarrow \infty} P(\alpha)=0$ (здесь $N$ - число элементов множества $S$ ).

3 а м е ч а н и е 4.2. Единственность решения уравнения (4.2) вытекает также из приведенной ниже теоремы 4.1. 
Обозначим через $D_{H}(F, \omega)$ хаусдорфову размерность множества $F \subset$ $S^{\mathrm{N}}$ относительно метрики $\rho_{\omega} ; \widehat{D}_{\mathscr{C}}(F, \omega)$ и $d_{\mathscr{C}, \mu_{\omega}}(x, \omega)$ - случайные аналоги величин $\widehat{D}_{\mathscr{C}}(F)$ и $d_{\mathscr{C}, \mu}(x)$, определенных в $\S 1$.

Лемма 4.6. Предположим, что с Q-вероятностью 1

$$
\limsup _{n \rightarrow \infty} \lambda\left(x_{n} ; \omega_{0}\right)<1, \quad x \in \Lambda_{\omega}
$$

Пусть $\alpha_{0}$ - решение уравнения $(4.2) ;$ тогда с Q-вероятностью 1

$$
d_{\mathscr{C}, \mu_{\omega}^{\left(\alpha_{0}\right)}}(x, \omega)=\lim _{n \rightarrow \infty} \frac{\ln \left[\mu_{\omega}^{\left(\alpha_{0}\right)}\left(C_{n}(x)\right)\right]}{\ln \left[a_{n}(x ; \omega)\right]}=\alpha_{0}
$$

для любого $x \in \Lambda$.

Д ок а з а те ль с т в о. Из условия (4.3) вытекает, что с $Q$-вероятностью 1 при любом $x \in \Lambda_{\omega}$ имеем $\limsup _{n \rightarrow \infty} n^{-1} \ln \left[a_{n}(x ; \omega)\right]<0$, и поэтому

$$
\lim _{n \rightarrow \infty} \frac{\ln \left[\mu_{\omega}^{\left(\alpha_{0}\right)}\left(C_{n}(x)\right)\right]}{\ln \left[a_{n}(x ; \omega)\right]}=\alpha_{0}-\lim _{n \rightarrow \infty} \frac{n^{-1} \ln \left[Z_{n}^{\left(\alpha_{0}\right)}(\omega)\right]}{n^{-1} \ln \left[a_{n}(x ; \omega)\right]}=\alpha_{0}
$$

Лемма доказана.

Разумеется, условие (4.3) выполнено, если еss $\sup _{\omega_{0} \in \Omega_{0}} \lambda\left(s ; \omega_{0}\right)<1$ при всех $s \in S$.

Из теоремы 2.1 и лемм 4.1 и 4.6 вытекает следующий результат.

Теорема 4.1. Пусть $\rho_{\omega}-$ случайная маситабная метрика; $\alpha_{0}$ - решение уравнения (4.2). Если выполнены условия (4.1) и (4.3), mо с Q-вероятностью $1 D_{H}\left(\Lambda_{\omega}, \omega\right)=\widehat{D}_{\mathscr{C}}\left(\Lambda_{\omega}, \omega\right)=D_{\mathscr{C}}\left(\Lambda_{\omega}, \omega\right)=\alpha_{0}$.

3 а м е ч а н и е 4.3 . В случае, когда $\lambda\left(s ; \omega_{1}\right) \equiv \lambda(s), s \in S$, уравнение (4.2) преврашается в уравнение Морана $\sum_{s \in S} \lambda^{\alpha}(s)=1$.

\section{§5. Хаусдорфова размерность фракталов в общих метрических пространствах}

В этом параграфе мы обобщим результаты 33 и $\$ 4$ на общие метрические пространства. В первой части рассматриваются фракталы, определяемые детерминированными конструкциями с конечной памятью; во второй части - фракталы, определяемые случайными конструкциями без памяти.

5.1. Детерминированные фракталы. Пусть $(M, \rho)$ - полное метрическое пространство; обозначим через $|A|-$ диаметр множества $A$ и через $[A]$ - его замыкание. Пусть $S=\{1, \ldots, N\}(N \in \mathbf{N})$; $S^{*}=\cup_{n=1}^{\infty} S^{n}$. Если дана последовательность $\mathbf{i}=\left(i_{1}, \ldots, i_{n}, \ldots\right) \in S^{\mathbf{N}}$, то $\mathbf{i}_{n} \stackrel{\text { def }}{=}\left(i_{1}, \ldots, i_{n}\right)$. Очевидно, $\mathbf{i}_{n} \in S^{n}$ и, обратно, любой вектор $\mathbf{i}^{*} \in S^{n}$ совпадает с сужением $\mathrm{i}_{n}$ некоторой последовательности $\mathrm{i} \in S^{\mathrm{N}}$; поэтому мы иногда обозначаем через $\mathbf{i}_{n}$ или через $\mathbf{j}_{n}$ любой произвольно фиксированный вектор из $S^{n}$; надеемся, что это не вызовет недоразумений. 
Рассмотрим в $S^{*}$ частичное упорядочение: для любого $\mathbf{i} \in S^{\mathbf{N}}$ положим $\mathbf{i}_{k}<\mathbf{i}_{n}$, если $k<n$.

Пусть дано семейство множеств $\left\{A_{\mathbf{i}^{*}}, \mathbf{i}^{*} \in S^{*}\right\}$. Обозначим $\widetilde{A}_{\mathbf{i}^{*}}=$ $\cup_{\mathbf{j}^{*} \mathbf{i}^{*}} A_{\mathbf{j}^{*}}$. Ясно, что при любом $\mathbf{i} \in S^{\mathbf{N}}$ последовательность $\left\{\tilde{A}_{\mathbf{i}_{n}}, n \in \mathbf{N}\right\}$ убывает. Семейство множеств $\left\{A_{\mathbf{i}^{*}}, \mathbf{i}^{*} \in S^{*}\right\}$ называется конструкцией, если $\lim _{n \rightarrow \infty}\left|\tilde{A}_{\mathbf{i}_{n}}\right|=0$ при любом $\mathrm{i} \in S^{\mathbf{N}}$.

Рассмотрим произвольную конструкцию $\left\{A_{\mathbf{i}^{*}}, \mathbf{i}^{*} \in S^{*}\right\}$. При любой фиксированной последовательности $\mathrm{i} \in S^{\mathbf{N}}$ мы выберем элементы $x_{\mathbf{i}_{n}} \in \widetilde{A}_{\mathbf{i}_{n}}, n \in \mathbf{N}$. Поскольку пространство $(M, \rho)$ полно, предел $\lim _{n \rightarrow \infty} x_{\mathbf{i}_{n}}$ существует и не зависит от выбора элементов $x_{i_{n}}$ в множестве $\tilde{A}_{\mathbf{i}_{n}}$, поэтому он полностью определяется последовательностью $\mathrm{i}$; мы обозначим этот элемент через $x_{\mathbf{i}}$. В результате этой процедуры мы получаем «фрактал» $F_{\{A\}}$, определяемый конструкцией $\left\{A_{\mathbf{i}^{*}}\right\}$ : это множество всех элементов $x_{\mathrm{i}}, \mathrm{i} \in S^{\mathbf{N}}$. Легко убедиться, что множество $F_{\{A\}}$ замкнуто и $F_{\{A\}}=\bigcap_{n=1}^{\infty} \cup_{i_{n} \in S^{n}}\left[\widetilde{A}_{\mathbf{i}_{n}}\right]$.

Будем говорить, что конструкции $\left\{A_{\mathbf{j}^{*}}\right\}$ и $\left\{D_{\mathbf{i}^{*}}\right\}$ конъюнктивны, если $A_{\mathrm{i}^{*}} \cap D_{\mathrm{i}^{*}} \neq \varnothing$ при любом $\mathrm{i}^{*} \in S^{*}$. Ясно, что конъюнктивные конструкиии определяют один и тот же фрактал.

Как и в $\S 3$, фиксируем натуральные числа $m$ и $n_{0} \geqslant m$ и рассмотрим в $S^{\mathbf{N}}$ множество $\Lambda_{0}$ и функции масштаба $\lambda(i)$ при $m=0$ и $\lambda\left(i \mid i_{1}, \ldots, i_{m}\right)$ при $m>0$ со значениями в интервале $[0,1) ;$ при любом $\mathbf{i}=\left(i_{1}, \ldots, i_{n}, \ldots\right)$ обозначим:

$$
\begin{aligned}
a_{n}(\mathbf{i}) & =\prod_{j=n_{0}+1}^{n} \lambda\left(i_{j}\right), \quad \text { если } n>n_{0}, m=0 ; \\
a_{n}(\mathbf{i}) & =\prod_{j=n_{0}+1}^{n} \lambda\left(i_{j} \mid i_{j-m}, \ldots, i_{j-1}\right), \text { если } n>n_{0}, m>0 ; \\
\Lambda & =\left\{\mathbf{i}: a_{n}(\mathbf{i})>0, n \in \mathbf{N}\right\} \cap \Lambda_{0} ; \quad \Lambda_{n}=\left\{\mathbf{i}: a_{n}(\mathbf{i})>0\right\} \cap \Lambda_{0}, \quad n>n_{0} .
\end{aligned}
$$

Предположим, что $A_{\mathbf{i}_{n}}=\varnothing$, коль скоро $\mathbf{i} \notin \Lambda_{n} ;$ иными словами, если $\mathrm{i} \notin \Lambda_{n}$, то множество $A_{\mathbf{i}_{n}}$ исключается из конструкции.

Для любого $n>n_{0}$ и для любого вектора $\mathbf{i} \in \Lambda_{n}$ мы рассмотрим в $F_{\{A\}}$ множество $\Gamma_{n}(\mathbf{i}) \stackrel{\text { def }}{=}\left\{x_{\mathbf{j}}: \mathbf{j}_{n}=\mathbf{i}_{n}\right\} ;$ рассмотрим также «кодирующее» отображение $\kappa: F \rightarrow \Lambda \subset S^{\mathbf{N}}$, определенное следующим образом: $\kappa\left(x_{\mathbf{i}}\right)=$ $\left(i_{1}, i_{2}, \ldots\right)$.

Нетрудно убедиться в справедливости следующего утверждения.

Лемма 5.1. Предположим, что конструкция $\left\{A_{\mathrm{i}^{*}}\right\}$ удовлетворяет следующему условию:

(СлО) Слабая отделимость: $п р и$ любом $n>n_{0}\left[\widetilde{A}_{\mathbf{i}_{n}}\right] \cap\left[\widetilde{A}_{\mathbf{j}_{n}}\right] \cap F_{\{A\}}=$ $\left(\left[\tilde{A}_{\mathbf{i}_{n}}\right] \cap F_{\{A\}}\right) \cap\left(\left[\tilde{A}_{\mathbf{j}_{n}}\right] \cap F_{\{A\}}\right)=\varnothing$, коль скоро $\mathbf{i}, \mathbf{j} \in \Lambda_{n} u \mathbf{i}_{n} \neq \mathbf{j}_{n}$.

Tozda: 

$\mathbf{i}, \mathbf{j} \in \Lambda_{n}$

1) отображение $\kappa$ взаимно однозначно; $п р и$ любых $n>n_{0} u$

2) $\Gamma_{n}(\mathbf{i})=F_{\{A\}} \cap\left[\tilde{A}_{\mathbf{i}_{n}}\right]$;

3) ecлu $\mathbf{i}_{n} \neq \mathbf{j}_{n}$, mo $\Gamma_{n}(\mathbf{i}) \cap \Gamma_{n}(\mathbf{j})=\varnothing$;

4) $F_{\{A\}}=U_{\mathbf{i} \in S} \mathbf{N} \Gamma_{n}(\mathbf{i})$;

5) $C_{n}(\mathbf{i}) \cap \Lambda=\kappa\left(\Gamma_{n}(\mathbf{i})\right)$.

3 а м е ч а н и е 5.1. Любое из следующих условий влечет условие ( $\mathrm{CлO})$ :

(О-1) Отделимость порядка 1: при любом $n>n_{0}\left[\tilde{A}_{\mathbf{i}_{n}}\right] \cap\left[\tilde{A}_{\mathbf{j}_{n}}\right]=\varnothing$, коль скоро $\mathbf{i}, \mathbf{j} \in \Lambda_{n}$ и $\mathbf{i}_{n} \neq \mathbf{j}_{n}$;

(О-2) Отделимость порядка 2: при любом $n>n_{0}\left[\tilde{A}_{\mathbf{i}_{n+1}}\right] \cap\left[\tilde{A}_{\mathbf{j}_{n+1}}\right]=$ $\varnothing$, коль скоро $\mathbf{i}, \mathbf{j} \in \Lambda_{n}$ и $\mathbf{i}_{n} \neq \mathbf{j}_{n}$.

Вообще, при любом $l \geqslant 2$ следующее условие влечет условие (СлО):

(О-l) Oтделимость порядка l: при любом $n\left[\widetilde{A}_{\mathbf{i}_{n+l-1}}\right] \cap\left[\widetilde{A}_{\mathbf{j}_{n+l-1}}\right]=\varnothing$, коль скоро $\mathbf{i}, \mathbf{j} \in \Lambda_{n}$ и $\mathbf{i}_{n} \neq \mathbf{j}_{n}$.

Обозначим через $\rho^{*}$ сужение метрики $\rho$ на $F_{\{A\}}$. Ясно, что $\tilde{\rho} \stackrel{\text { def }}{=}$ $\rho^{*} \circ \kappa^{-1}$ - метрика в $\Lambda$.

Лемма 5.2. $D_{H}\left(F_{\{A\}}\right)=D_{H}(\Lambda)$.

Д о к а з а т е л ь с т в о. Отображение $\kappa$ пространства $\left(F_{\{A\}}, \rho^{*}\right)$ на пространство $(\Lambda, \widetilde{\rho})$ изометрично.

Лемма 5.3. Пусть выполнены условия (СлО) $u$

(CC) Cкорость сжатия множеств $\tilde{A}_{\mathbf{i}_{n}}$ : ecлu $n>n_{0}$, i $\in \Lambda_{n} u$ $r>b_{1} a_{n}(\mathbf{i})$, mo $\tilde{A}_{\mathbf{i}_{n}} \subset B(z, r)$ при любом $z \in \widetilde{A}_{\mathbf{i}_{n}}$.

Тогда метрика $\widetilde{\rho}$ в $\Lambda$ удовлетворяет условию (ССЦ) из 2.

Д о к а з а т е л в с т в о. Из условия (СС) вытекает, что конструкция $\left\{\Gamma_{\mathbf{i}^{*}}\right\}$ в метрическом пространстве $\left(F_{\{A\}}, \rho^{*}\right)$ обладает следующим свойством: (СС') если $n \geqslant n_{0}, \mathbf{i} \in \Lambda_{n}$ и $r>b_{1} a_{n}(\mathbf{i})$, то $\Gamma_{n}(\mathbf{i}) \subset B\left(x_{\mathbf{i}}, r\right)$ при люобом $x_{\mathbf{i}} \in \Gamma_{n}(\mathbf{i})$.

Остается вспомнить, что $\kappa$ есть изометрия из $\left(F_{\{A\}}, \rho^{*}\right)$ на $(\Lambda, \widetilde{\rho})$ и что $\widetilde{C}_{n}(\mathbf{i})=\kappa\left(\Gamma_{n}(\mathbf{i})\right)$.

Теорема 5.1. Если выполнены условия (СлО) и (СС), то множество $F_{\{A\}}$ компактно.

Д о к а з а т е л ь т в о. Наше утверждение вытекает из леммы 5.3 и предложения 1.1 .

При других условиях компактность фракталов в $\mathbf{R}^{n}$ была установлена разными авторами (см., например, [12]).

Обобщим понятие индекса Морана, введенное в 1 (см. [22], [25]). Рассмотрим последовательность $\mathrm{i} \in S^{\mathrm{N}}$ и положительные числа $r$ и $b$. Если $b a_{n_{0}+1}(\mathbf{i}) \geqslant r$, определим натуральное число $l(r, \mathbf{i}, b)>n_{0}$ следуюшим образом: $b a_{l(r, \mathbf{i}, b)+1}(\mathbf{i})<r \leqslant b a_{l(r, \mathbf{i}, b)}(\mathbf{i}) ;$ если $b a_{n_{0}+1}(\mathbf{i})<r$, мы полагаем $l(r, \mathbf{i}, b)=n_{0}+1$. 
Индексом Морана конструкции $\left\{A_{\mathrm{i}^{*}}\right\}$, соответствуюшим постоянной $b$, называется минимальное число $L_{\left\{A_{\mathrm{i}^{*}}\right\}}(b)$, обладающее следующим свойством: для любого $x \in M$, любого $\mathrm{i} \in \Lambda$ и $n>n_{0}$ существует не более чем $L_{\left\{A_{\mathbf{i}^{*}}\right\}}(b)$ попарно непересекающихся множеств $\tilde{A}_{\mathbf{i}_{l\left(b a_{n}\right.}^{(k)}(\mathbf{i}) \mathbf{i}^{(k), b)}}$ таких, что $B\left(x, b a_{n}(\mathbf{i})\right) \cap\left[\tilde{A}_{\mathbf{i}_{l\left(b a_{n}(\mathrm{i}), \mathrm{i}^{(k)}, b\right)}}\right] \neq \varnothing ;$ если такое $L_{\left\{A_{\mathrm{i}^{*}}\right\}}(b)$ не сушествует, мы полагаем $L_{\left\{A_{\mathbf{i}^{*}}\right\}}(b)=\infty$.

Обозначим через $D_{H}\left(F_{\{A\}}\right)$ хаусдорфову размерность множества $F_{\{A\}}$. Пусть $\alpha_{0}$ - решение обобщенного уравнения Морана (3.2).

Теорема 5.2. Пусть существуют положительные числа $b_{1} \quad$ u $b_{2}$ такие, что конструкиия $\left\{A_{\mathrm{i}^{*}}, \mathrm{i}^{*} \in S^{*}\right\}$ или какая-либо конъюнктивная ей конструкиия удовлетворяет условиям (СлО), (СС) $и$

(КИМ) Конечность индекса Морана: $L_{\left\{A_{\mathrm{i}^{*}}\right\}}\left(b_{2}\right)<\infty$. Тогда $D_{H}\left(F_{\{A\}}\right)=\alpha_{0}$.

Д оказатель с т в о. Поскольку $\Gamma_{n}(\mathbf{i})=\left[\widetilde{A}_{\mathbf{i}_{n}}\right] \cap F_{\{A\}}$, то при любых $x^{*} \in F_{\{A\}}$ и $r>0$ из $B\left(x^{*}, r\right) \cap F_{\{A\}} \cap \Gamma_{n}(\mathrm{i}) \neq \varnothing$ вытекает $B\left(x^{*}, r\right) \cap\left[\tilde{A}_{\mathbf{i}_{n}}\right] \neq \varnothing$. Поэтому индекс Морана конструкции $\Gamma_{n}(\mathbf{i})$ в $\left(F_{\{A\}}, \rho^{*}\right)$ не больше индекса конструкции $\left\{A_{\mathbf{i}^{*}}\right\}$. Из этого и из леммы 5.3 вытекает, что метрика $\tilde{\rho}$ является масштабной метрикой в $\Lambda$. Остается применить лемму 5.2 и теорему 3.1 .

Разумеется, условие (КИМ) не отличается особенной наглядностью; обсудим варианты более сильных, но зато и более наглядных условий.

Теорема 5.3. Предположим, что конструкция $\left\{A_{\mathrm{i}^{*}}, \mathrm{i}^{*} \in S^{*}\right\}$ или какая-либо конъюнктивная ей конструкция удовлетворяет условию (СС) и существует положительная константа $b_{2}$ такая, что выполнено условие:

(СлРО) Слабая равномерная отделимость множеств $\tilde{A}_{\mathbf{i}_{n}}:$ при $n>n_{0}$

$$
\phi\left(\tilde{A}_{\mathbf{i}_{n}} \cap F_{\{A\}}, \tilde{A}_{\mathbf{j}_{n}} \cup F_{\{A\}}\right)>b_{2} \max \left(a_{n}(\mathbf{i}), a_{n}(\mathbf{j})\right)
$$

коль скоро $\mathbf{i}, \mathbf{j} \in \Lambda_{n} u \mathbf{i}_{n} \neq \mathbf{j}_{n}($ здесь $\phi(A, B)=\inf \{\rho(x, y): x \in A, y \in B\})$. Tогда $D_{H}\left(F_{\{A\}}\right)=\alpha_{0}$.

Д о к а з а т е ь с т в о. Из условий (СС) и (СлРО) вытекает, что $\widetilde{\rho}$ - масштабная метрика в $\Lambda$. Остается применить лемму 5.2 и теореMy 3.1 .

3 а м е ч а и е 5.2. При любом $l \geqslant 1$ следуюшее условие (РО- $l$ ) влечет условие (СлРО):

(РО-l) Равномерная отделимость порядка $l$ : при любом $n>n_{0}$ $\phi\left(\tilde{A}_{\mathbf{i}_{n+l-1}}, \widetilde{A}_{\mathbf{j}_{n+l-1}}\right)>b_{2} \max \left(a_{n}(\mathbf{i}), a_{n}(\mathbf{j})\right)$, коль скоро $\mathbf{i}, \mathbf{j} \in \Lambda_{n}$ и $\mathbf{i}_{n} \neq \mathbf{j}_{n}$.

Эти условия становятся слабее с увеличением $l$; они гораздо нагляднее, чем условие (СлРО), особенно при $l=1$ или 2 (см. ниже пример 5.3). 
Пусть $(M, \rho)$ - метрическое пространство; мы говорим, что $\rho$ удовлетворяет условию равномерной ограниченности упаковок шаров (РОУШ), если для каждого $a>1$ существует конечная постоянная $K(a)$ такая, что при любом $r>0$ всякий шар радиуса $a r$ содержит не более чем $K(a)$ непересекаюшихся шаров радиуса $r$.

II р и м е р 5.1. Пусть $M$ - нильпотентная группа Ли (например, $\left.\mathbf{R}^{m}\right) ; \rho$ - инвариантная метрика и $\mu$ - мера Хаара на $M$. Тогда для любого $a>>^{*} 1$ существует постоянная $C_{a}$ такая, что $\mu(B(x, a r)) \leqslant$ $C_{a} \mu(B(x, r))$ при любом $r>0$ (см. [17]). Метрика $\rho$ удовлетворяет $y$ словию (РОУІІ). Действительно, пусть шар $B(x, a r)$ содержит $k$ непересекаюпихся шаров $B\left(x^{(i)}, r\right)$; тогда при любом $x$ имеем: $k \mu(B(x, r))=$ $\mu\left(\cup_{i} B\left(x^{(i)}, r\right)\right) \leqslant \mu(B(x, a r)) \leqslant C_{a} \mu(B(x, r))$, и, следовательно, $K(a) \leqslant C_{a}$.

Теорема 5.4. Предположим, что метрика $\rho$ удовлетворяет условию (РОУІІІ). Пусть конструкиия $\left\{A_{\mathrm{j}^{*}}\right\}$ удовлетворяет условию (CC) с постоянной $b_{1}$, и пусть существует конъюнктивная конструкиия $\left\{D_{\mathbf{i}^{*}}, \mathbf{i}^{*} \in S^{*}\right\}$, состоящая из замкнутых шаров $D_{\mathrm{i}_{n}}=$ $\left[B\left(x_{\mathrm{i}_{n}}, b_{2} a_{n}(\mathrm{i})\right)\right]\left(b_{2}>0\right)$ и удовлетворяющая условию (СлО). Тогда $D_{H}\left(F_{\{A\}}\right)=\alpha_{0}$.

Доказател в с т о. Конструкция $\left\{D_{\mathbf{1}^{*}}\right\}$ удовлетворяет условию (СС) с постоянной $b_{1}^{\prime}=b_{1}+4 b_{2}$. Фиксируем постоянную $b>0$. Предположим, что при некотором $n>n_{0}$ существуют $x \in M$ и попарно непересекаюшиеся множества $\widetilde{D}_{\substack{\mathrm{i}^{(k)}\left(k a_{n}(\mathrm{i}), \mathrm{i}^{(k)}, b\right) \\ \text {. }}}, 1 \leqslant k \leqslant L$, такие, что $B\left(x, b a_{n}(\mathbf{i})\right) \cap \widetilde{D}_{\mathbf{i}_{l\left(b a_{n}(\mathbf{i}), \mathbf{i}^{(k)}, b\right)}} \neq \varnothing$. Ясно, что $\cup_{k=1}^{L} \widetilde{D}_{\mathbf{i}_{l\left(b a_{n}(\mathbf{i}), \mathbf{i}^{(k)}, b\right)}} \subset$ $B\left(x,\left(2 b_{1}^{\prime}+b\right) a_{n}(\mathbf{i})\right)$. Поскольку $b a_{n}(\mathbf{i}) \leqslant l\left(b a_{n}(\mathbf{i}), \mathbf{i}^{(k)}, b\right)$, то каждое множество $\widetilde{D}_{\mathbf{i}^{(k)}}$ содержит шар радиуса $b a_{n}(\mathbf{i})$. В силу условия (РОУШ), $L_{\{D\}}(b) \leqslant K\left(\left(2 b_{1}^{\prime}+b\right) / b\right)$. Наше утверждение следует из теоремы 5.2.

Следствие 5.1. Предположим, ито метрика $\rho$ удовлетворяет условию (РОУІІІ). Пусть конструкчия $\left\{A_{\mathrm{i}^{*}}\right\}$ удовлетворяет условиям (СлО) $u$ (СС), и пусть при некотором $b_{2}>0$ существуют иарь $B\left(x_{\mathrm{i}_{n}}, b_{2} a_{n}(\mathrm{i})\right) \subset \widetilde{A}_{\mathrm{i}_{n}}, \mathrm{i} \in S^{\mathbf{N}}, n \in \mathbf{N}$. Toгda $D_{H}\left(F_{\{A\}}\right)=\alpha_{0}$.

Это следствие обобщает и усиливает некоторые результаты из работ [22] и [25], относящиеся к фракталам в евклидовом пространстве при $k=0, m=0$.

ПІ р и м е р 5.2. Классическое канторово множество в $[0,1]$ представляет собой простейший пример фрактала, определяемого монотонной конструкцией, удовлетворяющей условиям (СС) и (РО-1).

П р и м е р 5.3. (Иерархическая система «планет» и «спутников».) Предположим, что все множества $A_{\mathbf{i}_{n}}$ попарно не пересекаются. Мы можем представить себе, что при $\mathrm{j}_{n}=\mathrm{i}_{n}$ каждое множество $A_{\mathrm{j}_{n+1}}$ является «спутником» «планеты» $A_{\mathbf{i}_{n}}$. Грубо говоря, условие (CC) означает, что 
на любом уровне $n$ нашей иерархии каждая «планета» $A_{\mathrm{i}_{n}}$ должна быть достаточно мала, а ее «спутники» должны быть близки к «планете» и на «порядок» меньше; условие (РО-2) запрешает, на любом уровне иерархии, «спутникам» разных «планет» приближаться слишком близко друг к другу; условие (О-2) запрешает «слипание» «спутников» разных «планет». Разумеется, мы можем выбрать точку $x_{\mathbf{i}_{n}}$ в множестве $A_{\mathbf{i}_{n}}$. Такие фракталы тесно связаны с орбитами интересных динамических систем (см. [4] и [5]).

5.2. Случайные фракталы. Пусть $(\Omega, \mathscr{F})$ - произведение счетного множества копий пространства $\left(\Omega_{0}, \mathscr{F}_{0}\right)$, и пусть $Q-$ эргодическая трансляционно инвариантная вероятностная мера на $\mathscr{F}$.

Рассмотрим множество $M$, оснашенное семейством метрик $\left\{\rho_{\omega}(x, y)\right.$, $\omega \in \Omega\}$; предположим, что для $Q$-почти всех $\omega \in \Omega$ метрическое пространство $\left(M, \rho_{\omega}\right)$ полно. Пусть любому $\omega \in \Omega$ соответствует конструкция $\left\{A_{\mathrm{i}^{*}}(\omega), \mathrm{i}^{*} \in S^{*}\right\}$; обозначим через $F_{\{A\}}(\omega)$ порожденный ею фрактал.

Как и в $\S 4$, рассмотрим случайные функции масштаба $\lambda\left(i ; \omega_{1}\right)$ со значениями в интервале $[0,1)$; при любом $\omega \in \Omega$ обозначим: $\Lambda_{\omega}=\{\mathbf{i}=$ $\left.\left(i_{1}, i_{2}, \ldots\right): \lambda\left(i_{n} ; \omega_{i_{n}}\right)>0, n \in \mathbf{N}\right\}$.

Пусть $\kappa_{\omega}$ - кодирующее отображение множества $F_{\{A\}}(\omega)$ на $\Lambda_{\omega}$; $\rho_{\omega}^{*}$ - сужение метрики $\rho_{\omega}$ на множество $F_{\{A\}}(\omega)$; рассмотрим также метрику $\tilde{\rho}_{\omega}=\rho_{\omega}^{*} \circ \kappa_{\omega}^{-1}$ в $\Lambda_{\omega}$. Разумеется, $\kappa_{\omega}$ - изометрический изоморфизм пространства $\left(F_{\{A\}}(\omega), \rho_{\omega}^{*}\right)$ на $\left(\Lambda_{\omega}, \tilde{\rho}_{\omega}\right)$. Этот изоморфизм позволяет свести изучение размерности фрактала $F_{\{A\}}(\omega)$ к фактам, установленным в $\S 4$ (см. лемму 5.2). Заметим, наконец, что если при любом $\omega \in \Omega$ конструкция $\left\{A_{\mathbf{i}^{*}}(\omega), \mathrm{i}^{*} \in S^{*}\right\}$ удовлетворяет условиям какойлибо из теорем $5.2,5.3,5.4$ или следствия 5.1 , то $\tilde{\rho}_{\omega}$ является случайной масштабной метрикой в $\Lambda_{\omega}$. Используя теорему 4.1 , мы приходим к следующему результату.

Теорема 5.5. Пусть выполнены условия (4.1) и (4.3), и пусть при любом $\omega$ метрика $\rho_{\omega}$ и множества $A_{\mathrm{i}_{n}}(\omega)$ удовлетворяют условиям какой-либо из теорем 5.2, 5.3, 5.4 или следствия 5.1. Тогда с Q-вероятностью 1 хаусдорфова размерность множества $F_{\{A\}}(\omega)$ относительно метрики $\rho_{\omega}$ равна $\alpha_{0}$ - решению уравнения (4.2).

При $M=\mathbf{R}^{n}$ сходные результаты были получены другим методом в работах [18] и [25].

\section{СПИСОК ЛИТЕРАТУРЫ}

1. Гантмахер Ф. Р. Теория матриц. М.: ГИТТЛ, 1953.

2. Корнфельд И. П., Синай Я.Г., Фомия С.В. Эргодическая теория. М.: Наука, 1980.

3. Халмош П. Теория меры. М.: ИЛ, 1953, 292 с. 
4. Afraimovich V., Zaslavsky G.M. Fractal and multifractal properties of exit times and Poincaré recurrences. - Phys. Rev. E(3), 1997, v. 55, № 5, part A, p. 5418-5426.

5. Afraimovich V., Zaslavsky G. M. Sticky orbits of chaotic Hamiltonian dynamics. Lecture Notes in Phys., 1998, v. 511, p. 59-82.

6. Billingsley $P$. Hausdorff dimension in probability theory. - Illinois J. Math., 1960, v. 4, p. 187-209.

7. Billingsley P. Hausdorff dimension in probability theory II. - Illinois J. Math., 1961 , v. 5 , p. $291-298$.

8. Билинеслей II. Эргодическая теория и информация. М.: Мир, 1969, 238 с.

9. Billingsley $P$. Probability and Measure. New York: Wiley, 1986.

10. Brin M., Katok A. On local entropy. - Lecture Notes in Math., 1981, v. 1007, p. 30-38.

11. Falconer $K$. Fractal Geometry: Mathematical Foundations and Applications. Chichester: Wiley, 1990, 288 p.

12. Falconer K. Random fractals. - Math. Proc. Cambridge Philos. Soc., 1986, v. 100, p. 559-582.

13. Falconer $K$. A subadditive thermodynamic formalism for mixing repellers. - J. Phys. A, 1988, v. 21, p. L737-L742.

14. Friedland Sh. Discrete Liapunov exponents and Hausdorff dimension. Preprint. Chicago: Univ. of Illinois, 1997.

15. Furstenberg $H$. Disjointness in ergodic theory, minimal sets, and a problem in Diophantine approximation. - Math. Systems Theory, 1967, v. 1, p. 1-49.

16. Graf S., Mauldin R.D., Williams S.C. The Exact Hausdorff Dimension in Random Recursive Constructions. Providence, RI: Amer. Math. Soc., 1988, 121 p.

17. Gromov $M$. Groups of polynomial growth and expanding maps. - Publ. Math. Inst. Hautes Etud. Sci., 1981, № 53, p. 53-78.

18. Kifer Yu. Fractals via random iterated function systems and random geometric constructions. - Fractal Geometry and Stochastics. Ed. by Ch. Bandt et al. Basel: Birkhäuser, 1995, p. 145-164.

19. Kifer Yu. Fractal dimensions and random transformations. - Trans. Amer. Math. Soc., 1996, v. 348, № 5, p. 2003-2038.

20. Mauldin R., Edgar G. Multifractal decomposition of digraph recursive fractals. Proc. London Math. Soc., 1992, v. 65, № 3, p. 604-628.

21. Mauldin R., Williams S. Random recursive constructions: Asymptotic geometric and topological properties. - Trans. Amer. Math. Soc., 1986, v. 295, p. 325-346.

22. Moran P. Additive functions of intervals and Hausdorff measure. - Proc, Cambridge Philos. Soc., 1946, v. 42, p. 15-23.

23. Pesin Ya. B. Dimension Theory in Dynamical Systems: Contemporary Views and Applications. Chicago/London: Univ. of Chicago Press, 1997.

24. Pesin Ya., Tempelman A. Correlation dimension of measures invariant under group actions. - Random Comput. Dynam., 1995, v. 3, p. 137-156.

25. Pesin Ya., Weiss H. On the dimension of deterministic and random Cantor-like sets, symbolic dynamics, and the Eckmann-Ruelle conjecture. - Comm. Math. Phys., 1996, v. 182, № 1, p. 105-153.

26. Rényi $A$. Dimension, entropy and information. - Trans. of the 2nd Prague Conference on Information Theory, Statistical Decision Functions, Random Processes (Liblice, 1959). New York: Academic Press, 1960, p. 545-556.

27. Young L.S. Dimension, entropy, and Lyapunov exponents. - Ergodic Theory Dynam. Systems, 1982, v. 2, p. 109-124. 